\title{
Vector Solitons of a Coupled Schrödinger System with Variable Coefficients
}

\author{
Juan Carlos Muñoz Grajales \\ Departamento de Matemáticas, Universidad del Valle, Calle 13, No. 100-00, Cali, Colombia \\ Correspondence should be addressed to Juan Carlos Muñoz Grajales; jcarlmz@yahoo.com
}

Received 26 April 2016; Accepted 26 June 2016

Academic Editor: Antonio Scarfone

Copyright ( 2016 Juan Carlos Muñoz Grajales. This is an open access article distributed under the Creative Commons Attribution License, which permits unrestricted use, distribution, and reproduction in any medium, provided the original work is properly cited.

We show the existence of waveforms of finite-energy (vector solitons) for a coupled nonlinear Schrödinger system with inhomogeneous coefficients. Furthermore, some of these solutions are approximated using a Newton-type iteration, combined with a collocation-spectral strategy to discretize the corresponding soliton equations. Some numerical simulations concerned with analysis of a collision of two oncoming vector solitons of the system are also performed.

\section{Introduction}

Several physical processes related to wave motion can be described using systems of coupled nonlinear Schrödinger (CNLS) equations. Recently there has been a great interest on the study of CNLS systems with nonlinear terms modulated by coefficients which depend either on space, time, or both. This research is motivated by the potential applications of these models to the fields of Bose-Einstein condensates (BECS) [1-8] and nonlinear optics [9-11]. For instance, in the case of BECS [12,13], a description of the effect of the Feshbach resonances in the mean field limit can be developed using CNLS systems of Gross-Pitaevskii equations [14, 15]. The study of wave propagation in Bose-Einstein twocomponent condensates with spatially inhomogeneous interactions has been a field of intense research activity in Physics in the last few years [16-24]. In particular, the investigation of multicomponent solitons (also known as vector solitons) has attracted a great deal of attention, starting with the classical work by Manakov [25]. This type of permanent finite-energy waveform arises in CNLS systems due to the interplay between the second-order dispersion and cubic or high-order nonlinearity.

The CNLS systems may also model beam propagation inside crystals, water wave interactions, biophysics [26], finance [27], and oceanography [28], and in the field of communications such equations have been employed to describe pulse propagation along orthogonal polarization axes in nonlinear optical fibers and in wavelength-divisionmultiplexing systems [29]. Further physical phenomena in nonlinear optics can be described by this family of equations (see [10] and references therein).

In this paper, we will consider theoretically the CNLStype system

$$
\begin{aligned}
& i \frac{\partial u}{\partial \xi}+i \delta \frac{\partial u}{\partial x}+K \frac{\partial^{2} u}{\partial x^{2}}+\sigma_{1} u+a(x)|u|^{2} u+b(x)|v|^{2} u \\
& +e(x) v^{2} u^{*}=0 \\
& i \frac{\partial v}{\partial \xi}-i \delta \frac{\partial v}{\partial x}+K \frac{\partial^{2} v}{\partial x^{2}}+\sigma_{2} v+c(x)|v|^{2} v+d(x)|u|^{2} v \\
& \quad+e(x) u^{2} v^{*}=0
\end{aligned}
$$

where $x \in \mathbb{R}$ and $\xi>0$. Here $u^{*}$ denotes the complex conjugate of $u$. We suppose that the coefficients $a, b, c, d, e$ are positive and depend only on the variable $x$ in order to focus on the influence of $x$-nonlinear modulations on the multicomponent solitons of the system. Furthermore $K, \delta$ are positive real constants. As mentioned above, such type of CNLS system can be used to model a variety of physical phenomena. In the case that all coefficients are constants, system (1) is a model to describe one-dimensional light 
propagation through a linearly birefringent lossless optical fiber, taking into account the Kerr effect (see Menyuk [30, 31], Agrawal [32], and Evangelides Jr. [33]). In applications to optical fibers, the variables $x, \xi$ in system (1) denote time and space, respectively, $\delta$ means the normalized strength of the linear birefringence ( $2 \delta$ is the inverse group velocity difference), and the model's parameters are usually constant or $\xi$-varying (i.e., they vary along the fiber axis). However, space-time-dependent parameters can be encountered in CNLS systems applied to BECS. Recently, Cardoso et al. [34] and Han et al. [35] used systems in the form of (1) with $\delta=0$ and $e \equiv 0$ to describe the interaction among the modes in one-dimensional Bose-Einstein condensates modulated in space and time. In this type of application, the variables $x$, $\xi$ denote space and retarded time, respectively, the functions $u=u(\xi, x), v=v(\xi, x)$ denote the complex envelopes of the propagating beam of the two modes, $\sigma_{1}$ and $\sigma_{2}$ are the external potentials, $K>0$ means the group velocity dispersion coefficient, and the physical parameters $a, b, c$, and $d$ (depending in this case on both $\xi$ and $x$ ) describe the strength of the cubic nonlinearities. The motivation of the study of the interaction of propagating waves in BoseEinstein condensates with spatial inhomogeneities comes from the discovery in the last years of novel experimental ways to control experimentally the interactions through optical manipulation of the Fechbach resonances [36].

The first aim of the present paper is to address the physically relevant question of the existence of vector solitons $(u, v)$ of system (1) with $\delta=0$ in the form

$$
\begin{aligned}
& u(\xi, x)=e^{i \alpha \xi} \widetilde{u}(x), \\
& v(\xi, x)=e^{i \alpha \xi} \widetilde{v}(x)
\end{aligned}
$$

and in the form

$$
\begin{aligned}
& u(\xi, x)=e^{i \alpha_{1} \xi-(i \delta / 2 K) x} \widetilde{u}(x), \\
& v(\xi, x)=e^{i \alpha_{2} \xi+(i \delta / 2 K) x} \widetilde{v}(x),
\end{aligned}
$$

for the case $e(x) \equiv 0$, where $\tilde{u}$ and $\widetilde{v}$ are positive real functions and $\alpha, \alpha_{1}$, and $\alpha_{2}$ are real constants.

It is important to point out that exact solutions of system (1) have been obtained only in particular cases. We mention the work by Belmonte-Beitia et al. [38], where explicit solutions of system (1) for $e \equiv 0$ and $\delta=0$ and some examples of variable coefficients were computed using Lie group theory. However, the integrations involved are very long and can not be evaluated as a closed form expression for general model's coefficients. In [39], Belmonte-Beitia et al. also proved the existence of vector solitons of the system above, in the case that the inhomogeneous coefficients $a, b$, $c$, and $d$ have compact support and $e \equiv 0$ and $\delta=0$. Furthermore, Kartashov et al. [11] computed numerically and analyzed the stability of two-component solitons in a medium with a periodic modulation of the nonlinear coefficients for system (1) with $e \equiv 0$ and $\delta=0$.

In the present paper, we wish to generalize the previous results by establishing analytically existence of vector solitons in forms (2) and (3), of full system (1), considering the extra cross-mode nonlinear terms preceded by the $x$-dependent coefficient $e(x)$, and a nonzero value of the parameter $\delta$. In the case of CNLS models for pulse propagation in optical fibers, this class of cross-mode terms account for the coherent nonlinear interaction between two linear polarizations of the electromagnetic waves. As pointed out by Menyuk [30], these modulation terms may play an important role in a fiber with very low birefringence. Recently, Muñoz Grajales and Quiceno [37] also illustrated the effect of these extra nonlinear terms and the parameter $\delta$ on modulation instability of a pulse along an optical fiber modelled by full system (1) but with constant coefficients. On the other hand, in [40], some analytical vector bright solitons were calculated for a generalized CNLS system to model BECS including self-phase modulation, cross-phase modulation coefficients, a time-dependent anti-trapping parabolic potential, and fourwave mixing nonlinear terms in the forms $v^{2} u^{*}$ and $u^{2} v^{*}$ with a time-dependent coefficient.

To study existence of vector solitons of system (1), we apply the positive operator theory introduced originally by Krasnosel'skii [41, 42], following the ideas by Benjamin et al. [43] in the framework of solitary wave solutions of a family of scalar dispersive models for water wave propagation.

In second place, we compute numerically some vector solitons of system (1) in forms (2) and (3) using a Newton iteration, combined with a collocation-spectral strategy to discretize the corresponding soliton equations. This strategy allows us to compute approximations to new vector solitons of the system for a variety of inhomogeneous model's coefficients. Some numerical simulations concerned with the collision of two oncoming vector solitons of the CNLS system are also performed.

The rest of this paper is organized as follows. In Section 2, we review some known results on fixed points of positive operators in a Fréchet space, necessary in order to develop the existence theory of vector solitons of system (1). In Section 3 we reduce the problem to find a fixed point of a nonlinear positive operator defined on a cone in an appropriate Fréchet space. In Section 4, we use the theory of fixed point index and positive operators to establish the existence of a family of solitons of system (1). In Section 5, we introduce the numerical solver employed to compute solitons of the system and illustrate the theoretical results. Finally, Section 6 contains the conclusions of our work.

\section{Preliminary Results}

In this section we include a brief review of some results from the functional analysis of positive operators whose domain constitutes a subset of a Fréchet space, following the papers by Benjamin et al. [43] and Chen et al. [44, 45]. We must recall that a Fréchet space $X$ is a metrizable and complete, locallyconvex, linear topological space (over the real numbers). On $X$ a sequence $\left(p_{n}\right)_{n}$ of seminorms can be defined in such a way that $p_{n+1}(x) \geq p_{n}(x)$ for every $x \in X$ and every $n=1,2,3, \ldots$ and that the formula

$$
d(x, y)=\sum_{j=1}^{\infty} \frac{1}{2^{j}}\left(\frac{p_{j}(x-y)}{1+p_{j}(x-y)}\right), \quad x, y \in X,
$$


provides a metric that generates a topology that coincides with the original topology on $X$. In this case, we say that $X$ is a Fréchet space with generating family of seminorms $\left(p_{n}\right)_{n}$. Hereafter, we use the notation

$$
\begin{aligned}
& \mathscr{B}_{r}=\{x \in X: d(x, 0)<r\}, \\
& \mathscr{B}_{r}^{j}=\left\{x \in X: p_{j}(x)<r\right\}, \quad j \in \mathbb{N} .
\end{aligned}
$$

It is clear from (4) that we have that $X=\mathscr{B}_{1}$. In general, a set $B$ in a topological linear space $X$ is said to be bounded if, for any neighborhood $U$ of 0 in $X$, there is $\lambda>0$ such that $\lambda B \subset U$. In the case of a Fréchet space with metric $d$ given by (4), a set $B$ in $X$ is bounded if and only if corresponding to each positive integer $j$ there is $R>0$ such that $B \subset \mathscr{B}_{R}^{j}$. If $r>0$, then $\mathscr{B}_{r}$ is usually not bounded (for details see [46]). A closed subset $K$ of a Fréchet space $X$ is a cone if the following conditions hold true:

$$
\begin{aligned}
\lambda K & =\{\lambda u: u \in K\} \subset K, \quad \forall \lambda \geq 0, \\
K+K & =\{u+v: u, v \in K\} \subset K, \\
K \cap\{-K\} & =K \cap\{-u: u \in K\}=\{0\} .
\end{aligned}
$$

From (6), $K$ must be convex. On the other hand, we also have a partial ordering on $K$ given by

$$
\begin{gathered}
x \prec y \Longleftrightarrow \\
y-x \in K .
\end{gathered}
$$

For any $0<r<R<\infty$, let us denote

$$
\begin{aligned}
K_{r} & =K \cap B_{r}, \\
\partial K_{r} & =K \cap \partial B_{r}, \\
K_{r}^{R} & =\{u \in K: r<d(u, 0)<R\} .
\end{aligned}
$$

An operator $\mathscr{A}$ defined on $K$ is said to be positive, if $\mathscr{A}(K) \subset$ $K$. On the other hand, we say that a positive operator $\mathscr{A}$ on $K$ is $K$-compact, if the set $\mathscr{A}\left(K_{r}\right)$ has a compact closure, for each $0 \leq r<1$. A triplet $(K, \mathscr{A}, U)$ is said to be admissible, if

(1) $K$ is a convex subset of $X$,

(2) $U \subset K$ is open in the relative topology on $K$,

(3) $\mathscr{A}$ is continuous and $K$-compact,

(4) there are no fixed points of $\mathscr{A}$ on $\partial U$, the boundary of the open set $U$ in the relative topology on $K$.

From Granas' work [47], there is an integer-valued function $i(K, \mathscr{A}, U)$ that satisfies the basic axioms of a fixed point index. Among them, we consider the following ones:

(i) Homotopy Invariant. If $(K, \mathscr{A}, U)$ and $(K, \mathscr{B}, U)$ are two admissible triplets and the operator $\mathscr{A}$ is homotopic to the operator $\mathscr{B}$ on $U$, then $i(K, \mathscr{A}, U)=$ $i(K, \mathscr{B}, U)$.

(ii) The Fixed Point Property. If $(K, \mathscr{A}, U)$ is admissible and $i(K, \mathscr{A}, U) \neq 0$, then $\mathscr{A}$ has at least one fixed point in $U$. (iii) Index of Constant Maps. If $(K, \mathscr{A}, U)$ is admissible and $\mathscr{A}$ is constant (i.e., there is a point $a \in K$ such that $\mathscr{A} u=a$ for all $u \in K)$, then

$$
i(K, \mathscr{A}, U)= \begin{cases}1 & \text { if } a \in U, \\ 0 & \text { if } a \notin U\end{cases}
$$

We refer the reader to [43] (see also [41, 42, 47]) for details in the following results. It is assumed throughout that $K$ is a cone in a Fréchet space with generating family of seminorms $\left(p_{j}\right)_{j}$ and the standard metric $d$ as in (4) and that $\mathscr{A}: K \rightarrow K$ is continuous, positive, and $K$-compact.

Lemma 1. Suppose that $0<\rho<1$ and either

$$
\begin{aligned}
& \mathscr{A} x-x \notin K, \quad \forall x \in \partial K_{\rho} \text { or } \\
& t \mathscr{A} x \neq x \quad \forall x \in \partial K_{\rho} \text { and all } t \in[0,1] .
\end{aligned}
$$

Then one has that $\left(K, \mathscr{A}, K_{\rho}\right)$ is admissible and $i\left(K, \mathscr{A}, K_{\rho}\right)=$ 1.

Lemma 2. Suppose that $0<\rho<1$ and either

$$
x-\mathscr{A} x \notin K, \quad \forall x \in \partial K_{\rho} \text { or }
$$

There exists $\tilde{x} \neq 0$ in $K$ such that

$$
x-\mathscr{A} x \neq \lambda \tilde{x} \quad \text { for } x \in \partial K_{\rho}, \lambda \geq 0 .
$$

Then $\left(K, \mathscr{A}, K_{\rho}\right)$ is admissible and $i\left(K, \mathscr{A}, K_{\rho}\right)=0$.

The following theorem is a consequence of the first two lemmas.

Theorem 3. If either (10) or (11) holds for $r$ satisfying $0<r<1$ and either (12) or (13) holds for $R$ satisfying $0<r<R<1$, then $\mathscr{A}$ has at least one fixed point in $K_{r}^{R}$. Moreover, $i\left(K, \mathscr{A}, K_{r}^{R}\right)=$ -1 .

An interesting property of system (1), allowing finding new solutions, is described in the following result.

Theorem 4 (Galilean invariance). Let $\delta=0$ and let $a, b, c, d$, $e, \sigma_{1}, \sigma_{2}$, and $K$ be constants. If $u(\xi, x)$ and $v(\xi, x)$ are a solution to system (1), then another solution is given by

$$
\begin{aligned}
& \tilde{u}(\xi, x)=u(\xi, x-V \xi) e^{i \alpha x} e^{-i K \alpha^{2} \xi}, \\
& \widetilde{v}(\xi, x)=v(\xi, x-V \xi) e^{i \alpha x} e^{-i K \alpha^{2} \xi},
\end{aligned}
$$

where the velocity $V$ is given by

$$
V=2 \alpha K
$$

Furthermore, the solutions of system (1) are indifferent to multiplication by $e^{i \Phi}$, for any constant $\Phi$.

Proof. It follows directly by substitution into system (1). 


\section{Problem Setting}

In the present paper, we are interested in establishing the existence of vector solitons $(u, v)$ of system (1) for $\delta=0$ in the form

$$
\begin{aligned}
& u(\xi, x)=e^{i \alpha \xi} \widetilde{u}(x), \\
& v(\xi, x)=e^{i \alpha \xi} \widetilde{v}(x)
\end{aligned}
$$

and in the form

$$
\begin{aligned}
& u(\xi, x)=e^{i \alpha_{1} \xi-(i \delta / 2 K) x} \widetilde{u}(x), \\
& v(\xi, x)=e^{i \alpha_{2} \xi+(i \delta / 2 K) x} \widetilde{v}(x),
\end{aligned}
$$

for the case $e(x) \equiv 0$. Here $\tilde{u}$ and $\widetilde{v}$ are positive real functions, and $\alpha, \alpha_{1}$, and $\alpha_{2}$ are real constants. In each case, we see that the functions $\widetilde{u}$ and $\widetilde{v}$ must satisfy a system in the form (soliton equations)

$$
\begin{aligned}
& \left(I-\beta_{1} \partial_{x}^{2}\right) \tilde{u}=A(x) \tilde{u}^{3}+B(x) \tilde{v}^{2} \tilde{u}, \\
& \left(I-\beta_{2} \partial_{x}^{2}\right) \tilde{v}=C(x) \widetilde{v}^{3}+D(x) \tilde{u}^{2} \widetilde{v},
\end{aligned}
$$

where the coefficients $A(x), B(x), C(x), D(x), \beta_{1}$, and $\beta_{2}$ are defined in the case that $\delta=0$ by

$$
\begin{gathered}
\beta_{1}=\frac{K}{\alpha-\sigma_{1}}, \\
\beta_{2}=\frac{K}{\alpha-\sigma_{2}}, \\
A(x)=\frac{a(x)}{\alpha-\sigma_{1}}, \\
B(x)=\frac{b(x)+e(x)}{\alpha-\sigma_{1}}, \\
C(x)=\frac{c(x)}{\alpha-\sigma_{2}}, \\
D(x)=\frac{d(x)+e(x)}{\alpha-\sigma_{2}} .
\end{gathered}
$$

On the other hand, when $e \equiv 0$,

$$
\begin{array}{r}
\beta_{1}=\frac{K}{\alpha_{1}-\sigma_{1}-\delta^{2} / 4 K}, \\
\beta_{2}=\frac{K}{\alpha_{2}-\sigma_{2}-\delta^{2} / 4 K}, \\
A(x)=\frac{a(x)}{\alpha_{1}-\sigma_{1}-\delta^{2} / 4 K}, \\
B(x)=\frac{b(x)}{\alpha_{1}-\sigma_{1}-\delta^{2} / 4 K}, \\
C(x)=\frac{c(x)}{\alpha_{2}-\sigma_{2}-\delta^{2} / 4 K}, \\
D(x)=\frac{d(x)}{\alpha_{2}-\sigma_{2}-\delta^{2} / 4 K} .
\end{array}
$$

We assume that the coefficients $a(x), b(x), c(x), d(x)$, and $e(x)$ in system (1) are bounded, continuous, positive, even, and nonincreasing for $x \geq 0, K>0$, the parameters $\delta, \sigma_{1}, \sigma_{2}, \alpha$, $\alpha_{1}$, and $\alpha_{2}$ are such that the coefficients $A(x), B(x), C(x)$, and $D(x)$ are positive and bounded, and $\beta_{1}$ and $\beta_{2}$ are positive.

Abandoning the tildes, we see that to show the existence of a solution $U=(u, v)^{t}$ of $(18)$ is equivalent to establish the existence of a solution of the fixed point equation:

$$
U=\mathscr{A}(U)
$$

where the operator $\mathscr{A}$ is defined by

$$
\begin{aligned}
\mathscr{A}(U) & =\left(\begin{array}{l}
\mathscr{A}_{1}(U) \\
\mathscr{A}_{2}(U)
\end{array}\right) \\
& =\left(\begin{array}{c}
\left(I-\beta_{1} \partial_{x}^{2}\right)^{-1}\left(A u^{3}+B v^{2} u\right) \\
\left(I-\beta_{2} \partial_{x}^{2}\right)^{-1}\left(C v^{3}+D u^{2} v\right)
\end{array}\right) .
\end{aligned}
$$

Let us denote by $\hat{f}$ the Fourier transform of the function $f$. We define the functions $K_{1}$ and $K_{2}$ as

$$
\begin{aligned}
& \widehat{K}_{1}(y)=\frac{1}{1+\beta_{1} y^{2}}, \\
& \widehat{K}_{2}(y)=\frac{1}{1+\beta_{2} y^{2}} .
\end{aligned}
$$

Thus $\widehat{K}_{1}$ and $\widehat{K}_{2}$ are positive, even, and monotone decreasing on $(0, \infty)$ and belong to $L^{1}(\mathbb{R})$. Furthermore for $i=1,2$,

$$
K_{i}(x)=\frac{2}{2 \pi} \int_{0}^{\infty} \frac{\cos (x y)}{1+\beta_{i} y^{2}} d y=\frac{e^{-\beta_{i}^{-1 / 2}|x|}}{2 \beta_{i}^{1 / 2}} .
$$

Therefore $K_{1}, K_{2} \in C(\mathbb{R}) \cap L^{1}(\mathbb{R})$.

Then we can rewrite the operator $\mathscr{A}$ as

$$
\mathscr{A}(U)=\left(\begin{array}{l}
\mathscr{A}_{1}(U) \\
\mathscr{A}_{2}(U)
\end{array}\right)=\left(\begin{array}{l}
K_{1} *\left(A u^{3}+B v^{2} u\right) \\
K_{2} *\left(C v^{3}+D u^{2} v\right)
\end{array}\right),
$$

where $f * g$ denotes the convolution between the functions $f$ and $g$.

Hereafter, we consider the space of real valued continuous functions defined on $\mathbb{R}$ (denoted by $C(\mathbb{R})$ ), with the topology of uniform convergence on bounded intervals under the seminorms

$$
p_{j}(w)=\max _{-j \leq x \leq j}|w(x)|, \quad j=1,2, \ldots
$$

In this case, the distance is given by

$$
\begin{aligned}
d(f, g) & =\sum_{j=1}^{\infty} \frac{1}{2^{j}}\left(\frac{d_{j}(f, g)}{1+d_{j}(f, g)}\right), \\
d_{j}(f, g) & =\sup _{x \in[-j, j]}|f(x)-g(x)| .
\end{aligned}
$$


The open ball of radius $r<1$ centered at zero and its boundary are given, respectively, by

$$
\begin{gathered}
B_{r}(0)=\{u \in C(\mathbb{R}): d(0, u)<r\}, \\
\partial B_{r}(0)=\{u \in C(\mathbb{R}): d(0, u)=r\} .
\end{gathered}
$$

Let $\mathscr{K} \subset C(\mathbb{R})$ be the cone defined as

$$
\begin{aligned}
\mathscr{K} & =\{w \in C(\mathbb{R}): w(x)=w(-x) \\
& \geq 0 ; w \text { is nonincreasing for } x \geq 0\} .
\end{aligned}
$$

Note the that, for $j=1,2,3 \ldots$, we have for all $w \in \mathscr{K}$ that

$$
p_{j}(w)=p_{1}(w),
$$

and so we have for $0<r<1$ that

$$
\begin{aligned}
d(w, 0) & =\sum_{j=1}^{\infty} \frac{1}{2^{j}}\left(\frac{p_{j}(w)}{1+p_{j}(w)}\right)=\frac{w(0)}{1+w(0)}<r \Longleftrightarrow \\
w(0) & <\frac{r}{1-r} .
\end{aligned}
$$

We note that the product space $C(\mathbb{R}) \times C(\mathbb{R})$ is also a Fréchet space with generating family of seminorms

$$
P_{j}(f, g)=\max _{x \in[-j, j]}\{|f(x)|,|g(x)|\} .
$$

We also define the cone $\widetilde{\mathscr{K}} \subset C(\mathbb{R}) \times C(\mathbb{R})$ by $\widetilde{\mathscr{K}}=\mathscr{K} \times \mathscr{K}$. by

The metric in the product space $C(\mathbb{R}) \times C(\mathbb{R})$ is defined

$$
\widetilde{d}((f, g),(u, v))=\sum_{j=1}^{\infty} 2^{-j} \frac{P_{j}(f-u, g-v)}{1+P_{j}(f-u, g-v)}
$$

In particular, for $(f, g) \in \widetilde{\mathscr{K}}$,

$$
\begin{aligned}
\widetilde{d}((f, g), 0) & =\sum_{j=1}^{\infty} 2^{-j} \frac{P_{j}(f, g)}{1+P_{j}(f, g)} \\
& =\frac{\max \{f(0), g(0)\}}{1+\max \{f(0), g(0)\}} .
\end{aligned}
$$

We also set

$$
\begin{aligned}
\mathscr{B}_{r} & (0) \\
\quad & =\{(f, g) \in C(\mathbb{R}) \times C(\mathbb{R}): \tilde{d}((f, g), 0)<r\},
\end{aligned}
$$

and its boundary

$$
\begin{aligned}
& \partial \mathscr{B}_{r}(0) \\
& \quad=\{(f, g) \in C(\mathbb{R}) \times C(\mathbb{R}): \tilde{d}((f, g), 0)=r\} .
\end{aligned}
$$

We observe that if $(f, g) \in \widetilde{\mathscr{K}} \cap \partial \mathscr{B}_{r}(0)$, that is, $\tilde{d}((f, g), 0)=$ $r$, then we have that

$$
\max \{f(0), g(0)\}=\frac{r}{1-r} .
$$

We denote by $\widetilde{\mathscr{K}}_{r}^{R}$ the annular section of the cone $\widetilde{\mathscr{K}}$ :

$$
\widetilde{\mathscr{K}}_{r}^{R}=\{u \in \widetilde{\mathscr{K}}: r<\widetilde{d}(u, 0)<R\} .
$$

Hereafter, according to the notation introduced in the previous section, we set, for $r>0$, the convex set

$$
\widetilde{\mathscr{K}}_{r}=\widetilde{\mathscr{K}} \cap \mathscr{B}_{r}(0)
$$

\section{Main Results}

Before we go further, we establish a general result.

Lemma 5. Let $k \in C(\mathbb{R}) \cap L^{1}(\mathbb{R})$ be an even bounded positive function on $\mathbb{R}$, which is monotone decreasing on $(0, \infty)$. Then the operator $\mathscr{B}$ defined by

$$
\mathscr{B}(f)(x)=(k * f)(x)=\int_{\mathbb{R}} k(x-r) f(r) d r
$$

maps $\mathscr{K}$ into $\mathscr{K}$.

Proof. We first note that $\mathscr{B}(f)(x)$ is bounded due to the fact that $k \in L^{1}(\mathbb{R})$ and also from the Young inequality, since

$$
\max _{\mathbb{R}}|k * f| \leq f(0)\|k\|_{L^{1}(\mathbb{R})}, \quad f \in \mathscr{K} .
$$

Now, we also have that $\mathscr{B}(f) \geq 0$ for $f \in \mathscr{K}$. In fact,

$$
\mathscr{B}(f)(x)=\int_{\mathbb{R}} k(x-y) f(y) d y \geq 0,
$$

since $k \geq 0$ and $f \geq 0$. On the other hand, $\mathscr{B}(f)$ is also an even function for $f \in \mathscr{K}$. In fact,

$$
\begin{aligned}
\mathscr{B}(f)(-x) & =\int_{\mathbb{R}} k(-x-y) f(y) d y \\
& =\int_{\mathbb{R}} k(x+y) f(y) d y \\
& =\int_{\mathbb{R}} k(x-z) f(z) d z=\mathscr{B}(f)(x) .
\end{aligned}
$$

We claim now that $\mathscr{B}(f)$ for $f \in \mathscr{K}$ is a continuous function on $\mathbb{R}$. In fact, first note that $0 \leq f(y) \leq f(0)$ for any $y \in \mathbb{R}$ :

$$
\begin{aligned}
& |\mathscr{B}(f)(x+h)-\mathscr{B}(f)(x)| \\
& \quad \leq \int_{\mathbb{R}}|k(x+h-y) f(y)-k(x-y) f(y)| d y \\
& \quad \leq f(0) \int_{\mathbb{R}}|k(x+h-y)-k(x-y)| d y \\
& \quad \leq f(0) \int_{\mathbb{R}}|k(y+h)-k(y)| d y .
\end{aligned}
$$

Using $k \in L^{1}(\mathbb{R})$ and the dominated convergence theorem, we conclude that

$$
\lim _{h \rightarrow 0}|\mathscr{B}(f)(x+h)-\mathscr{B}(f)(x)|=0,
$$


meaning that $\mathscr{B}(f)$ is a continuous function on $\mathbb{R}$, as long as $f \in \mathscr{K}$. Finally, we need to establish that $\mathscr{B}(f)$ is a nonincreasing function for $x \geq 0$, for $f \in \mathscr{K}$. So let $f \in \mathscr{K}$ be fixed and take $x \geq 0$ and $h>0$. Then, we have for any $r \in \mathbb{R}$ that

$$
\begin{aligned}
\mathscr{B}(f)(x)= & \int_{\mathbb{R}} k(x-y) f(y) d y \\
= & \int_{-\infty}^{r} k(x-y) f(y) d y \\
& +\int_{r}^{\infty} k(x-y) f(y) d y \\
= & \int_{0}^{\infty} k(x-r-z) f(z+r) d z \\
& +\int_{0}^{\infty} k(x-r-z) f(z+r) d z,
\end{aligned}
$$

and so we have that

$$
\begin{aligned}
\mathscr{B}(f)(x+h)= & \int_{0}^{\infty} k(x+h-z-r) f(z+r) d z \\
& +\int_{0}^{\infty} k(x+h+z-r) f(z-r) d z .
\end{aligned}
$$

Using $r=-h / 2$ in the first formula and $r=h / 2$ in the second one, we get that

$$
\begin{aligned}
\mathscr{B} & (f)(x)-\mathscr{B}(f)(x+h) \\
= & \int_{0}^{\infty}\left(k\left(z-x-\frac{1}{2} h\right)-k\left(x+\frac{1}{2} h+z\right)\right) \\
& \cdot\left(f\left(z-\frac{1}{2} h\right)-f\left(z+\frac{1}{2} h\right)\right) d z .
\end{aligned}
$$

Now, we note that

$$
\begin{gathered}
\int_{x+(1 / 2) h}^{\infty}\left(k\left(z-x-\frac{1}{2} h\right)-k\left(x+\frac{1}{2} h+z\right)\right) \\
\cdot\left(f\left(z-\frac{1}{2} h\right)-f\left(z+\frac{1}{2} h\right)\right) d z \geq 0,
\end{gathered}
$$

since $z \geq x+(1 / 2) h \geq(1 / 2) h$, and the fact that $k$ and $f$ are nonincreasing for $w \geq 0$. Now, for the rest of the integral, we use a similar argument, after noting that $k$ and $f$ are even functions. In fact, first note for $z \geq 0$ that

$$
\begin{aligned}
& f\left(\frac{1}{2} h-z\right)-f\left(z+\frac{1}{2} h\right) \\
& =f\left(z-\frac{1}{2} h\right)-f\left(z+\frac{1}{2} h\right) \geq 0, \\
& \text { for either } z \geq \frac{1}{2} h \text { or } z \leq \frac{1}{2} h,
\end{aligned}
$$

since $f$ is an even nonincreasing function for $w \geq 0$. So, from this fact, we have that

$$
\begin{gathered}
\int_{0}^{x+(1 / 2) h}\left(k\left(x+\frac{1}{2} h-z\right)-k\left(x+\frac{1}{2} h+z\right)\right) \\
\cdot\left(f\left(\frac{1}{2} h-z\right)-f\left(z+\frac{1}{2} h\right)\right) d z \geq 0,
\end{gathered}
$$

since $0 \leq z \leq x+(1 / 2) h$, and the fact that $k$ is an even nonincreasing function for $w \geq 0$. In other words, we have shown that

$$
\mathscr{B}(f)(x)-\mathscr{B}(f)(x+h) \geq 0,
$$

for any $x \geq 0$ and $h>0$, which means that $\mathscr{B}(f)(x)$ is a nonincreasing function for $x \geq 0$.

Lemma 6. Suppose that the coefficients $A(x), B(x), C(x)$, and $D(x)$ in system (18) are continuous bounded positive nonzero functions in $\mathbb{R}$ and $\beta_{1}>0, \beta_{2}>0$. Furthermore, assume that these coefficients are even and nonincreasing for $x \geq 0$. Then the operator $\mathscr{A}$ defined by (25) maps continuously $\widetilde{\mathscr{K}}$ into $\widetilde{\mathscr{K}}$. For each $0<r<1$, the set $\mathscr{A}\left(\widetilde{\mathscr{K}}_{r}\right)$ is a relative compact subset of $\widetilde{\mathscr{K}}$.

Proof. As we mention above, $K_{1}, K_{2} \in L^{1}(\mathbb{R})$ are positive functions and also from the Young inequality

$$
\max _{\mathbb{R}}\left|K_{i} * f\right| \leq f(0)\left\|K_{i}\right\|_{L^{1}(\mathbb{R})}, \quad f \in \mathscr{K}, i=1,2
$$

If we set $\mathscr{B}_{i}(f)=K_{i} * f$, then from Lemma 5 , we have that $\mathscr{B}_{i}(f) \in \mathscr{K}$ for any $f \in \mathscr{K}$. In other words, $\mathscr{B}_{i}(f)$ is an even, continuous, positive, bounded function on $\mathbb{R}$. Further, $\mathscr{B}_{i}(f)$ is a nonincreasing function on $(0, \infty)$.

Now, let $(u, v) \in \widetilde{\mathscr{K}}$, then we have that $A u^{3}+B v^{2} u$ and $C v^{3}+D u^{2} v$ belong to the cone $\mathscr{K}$, which means from Lemma 5 that

$$
\begin{aligned}
& \mathscr{A}_{1}(u, v) \geq 0, \\
& \mathscr{A}_{2}(u, v) \geq 0
\end{aligned}
$$

and also that $\mathscr{A}(u, v)$ for $(u, v) \in \widetilde{\mathscr{K}}$ is continuous on $\mathbb{R}$. Moreover, we also have that $\mathscr{A}_{1}(u, v)$ and $\mathscr{A}_{2}(u, v)$ are nonincreasing for $x \geq 0$, for all $(u, v) \in \widetilde{\mathscr{K}}$. In other words, we have established that $\mathscr{A}(\widetilde{\mathscr{K}}) \subset \widetilde{\mathscr{K}}$.

Finally, we want to prove that the operator $\mathscr{A}=\left(\mathscr{A}_{1}, \mathscr{A}_{2}\right)$ maps $\widetilde{\mathscr{K}}$ continuously to $\widetilde{\mathscr{K}}$. To see this, we must recall that convergence in $(C(\mathbb{R}) \times C(\mathbb{R}), \tilde{d})$ is equivalent to uniform convergence on closed bounded intervals $I \subset \mathbb{R}$. Assume that $\left(u_{n}, v_{n}\right) \rightarrow(u, v)$ in $(C(\mathbb{R}) \times C(\mathbb{R}), \widetilde{d})$, as $n \rightarrow \infty$. Let $I=[a, b] \subset \mathbb{R}$ be a fixed closed bounded interval. For $\epsilon>0$ given, we choose $\Omega>0$ sufficiently large such that, for $i=1,2$,

$$
\int_{-\infty}^{b-\Omega} K_{i}(y) d y+\int_{a+\Omega}^{\infty} K_{i}(y) d r<\epsilon .
$$

Using this fact, we see easily for $i=1,2$,

$$
\begin{aligned}
\max _{x \in I} & \int_{|y| \geq \Omega} K_{i}(x-y) d y \\
& \leq \int_{-\infty}^{b-\Omega} K_{i}(y) d y+\int_{a+\Omega}^{\infty} K_{i}(y) d y<\epsilon .
\end{aligned}
$$


To analyze the continuity of the first component of the operator $\mathscr{A}$, we decompose the integral on $\mathbb{R}$ as

$$
\begin{aligned}
& \left|\mathscr{A}_{1}\left(u_{n}, v_{n}\right)(x)-\mathscr{A}_{1}(u, v)(x)\right| \leq \int_{|y| \leq \Omega} K_{1}(x-y) \\
& \cdot\left(A\left|u_{n}^{3}-u^{3}\right|+B\left|v_{n}^{2} u_{n}-v^{2} u\right|\right) d y \\
& +\int_{|y| \geq \Omega} K_{1}(x-y) \\
& \cdot\left(A\left|u_{n}^{3}-u^{3}\right|+B\left|v_{n}^{2} u_{n}-v^{2} u\right|\right) d y .
\end{aligned}
$$

Observe that $\left\{(u, v),\left(u_{n}, v_{n}\right), n \in \mathbb{N}\right\}$ is a bounded set in $(C(\mathbb{R}) \times C(\mathbb{R}), \widetilde{d})$. Thus there exists a constant $\widetilde{C}>0$ such that

$$
\max _{x \in \mathbb{R}}\left\{|u(x)|,|v(x)|,\left|u_{n}(x)\right|,\left|v_{n}(x)\right|\right\} \leq \widetilde{C} .
$$

Let us denote

$$
M:=\sup _{x \in \mathbb{R}}[A(x)+B(x)] .
$$

Therefore, for $x \in[a, b]$ and $\Omega$ large enough,

$$
\begin{aligned}
& \int_{|y| \geq \Omega} K_{1}(x-y)\left(A\left|u_{n}^{3}-u^{3}\right|+B\left|v_{n}^{2} u_{n}-v^{2} u\right|\right) d y \\
& \leq 2 M \widetilde{C}^{3} \int_{|y| \geq \Omega} K_{1}(x-y) d y \leq 2 M \widetilde{C}^{3} \frac{\epsilon}{4 M \widetilde{C}^{3}} \\
& \quad=\frac{\epsilon}{2} .
\end{aligned}
$$

On the other hand, using the factorizations

$$
\begin{aligned}
u_{n}^{3}-u^{3} & =\left(u_{n}-u\right)\left(u_{n}^{2}+u_{n} u+u^{3}\right), \\
v_{n}^{2} u_{n}-v^{2} u & =\left(v_{n}-v\right)\left(v_{n}+v\right) u_{n}+v^{2}\left(u_{n}-u\right),
\end{aligned}
$$

we have that

$$
\begin{aligned}
& \int_{|y| \leq \Omega} K_{1}(x-y)\left(A\left|u_{n}^{3}-u^{3}\right|+B\left|v_{n}^{2} u_{n}-v^{2} u\right|\right) d y \\
& \leq 3 \widetilde{C}^{2} \int_{|y| \leq \Omega} K_{1}(x-y) \\
& \cdot\left((A+B)\left|u_{n}-u\right|+B\left|v_{n}-v\right|\right) d y \leq 3 \widetilde{C}^{2} M \\
& \cdot \max _{y \in[-\Omega, \Omega]}\left\{\left|u_{n}(y)-u(y)\right|+\left|v_{n}(y)-v(y)\right|\right\} \\
& \cdot \int_{x \in \mathbb{R}} K_{1}(x) d x \leq 3 \widetilde{C}^{2} M \\
& \cdot \max _{y \in[-\Omega, \Omega]}\left\{\left|u_{n}(y)-u(y)\right|+\left|v_{n}(y)-v(y)\right|\right\} .
\end{aligned}
$$

Due to $\left(u_{n}, v_{n}\right) \rightarrow(u, v)$ in $(C(\mathbb{R}) \times C(\mathbb{R}), \widetilde{d})$ as $n \rightarrow \infty$, given $\epsilon>0$, there exists $n_{0} \in \mathbb{N}$ such that if $n \geq n_{0}$,

$$
\begin{gathered}
\max _{y \in[-\Omega, \Omega]}\left(\left|u_{n}(y)-u(y)\right|,\left|v_{n}(y)-v(y)\right|\right) \\
<\frac{\epsilon}{12 \widetilde{C}^{2} M} .
\end{gathered}
$$

Therefore, it follows that

$$
\begin{aligned}
& \int_{|y| \geq \Omega} K_{1}(x-y)\left(A\left|u_{n}^{3}-u^{3}\right|+B\left|v_{n}^{2} u_{n}-v^{2} u\right|\right) d y \\
& \quad \leq \frac{\epsilon}{2}
\end{aligned}
$$

Substituting the results above into inequality (57), it follows that

$$
\max _{x \in[a, b]}\left|\mathscr{A}_{1}\left(u_{n}, v_{n}\right)(x)-\mathscr{A}_{1}(u, v)(x)\right| \leq \epsilon
$$

for $n \geq n_{0}$. Analogously,

$$
\max _{x \in[a, b]}\left|\mathscr{A}_{2}\left(u_{n}, v_{n}\right)(x)-\mathscr{A}_{2}(u, v)(x)\right| \leq \epsilon,
$$

for $n \geq n_{0}$. We conclude that the operator $\mathscr{A}$ maps $\widetilde{\mathscr{K}}$ continuously to $\widetilde{\mathscr{K}}$.

It remains proving that $\mathscr{A}\left(\widetilde{\mathscr{K}}_{r}\right)(0<r<1)$ is relative compact subset of $\widetilde{\mathscr{K}}$, which is equivalent that $\mathscr{A}_{i}\left(\widetilde{\mathscr{K}}_{r}\right)$ is relative compact subset of $\widetilde{\mathscr{K}}$, for $i=1,2$. To see this, we use the Arzela-Ascoli Theorem to establish the compactness of the families $\mathscr{M}_{i}$ in $C(\mathbb{R})$,

$$
\begin{aligned}
\mathscr{M}_{1} & =\left\{w \in C(\mathbb{R}): w=\mathscr{B}_{1}\left(A u^{3}+B v^{2} u\right), \quad(u, v)\right. \\
& \left.\in \widetilde{\mathscr{K}}_{r}\right\} \\
\mathscr{M}_{2} & =\left\{w \in C(\mathbb{R}): w=\mathscr{B}_{2}\left(C v^{3}+D u^{2} v\right), \quad(u, v)\right. \\
& \left.\in \widetilde{\mathscr{K}}_{r}\right\} .
\end{aligned}
$$

Let $(u, v) \in \widetilde{\mathscr{K}}_{r}$ be such that $w=\mathscr{B}_{i}\left(f_{i}\right)$ with $f_{1}=A u^{3}+B v^{2} u$ and $f_{2}=C v^{3}+D u^{2} v$. We first note that, for $f_{i} \in C(\mathbb{R})$, we have that $\mathscr{B}_{i}\left(f_{i}\right): \mathbb{R} \rightarrow \mathbb{R}$ is a continuous function such that (see Lemma 5)

$$
\begin{aligned}
& \left|\mathscr{B}_{i}(f)(x+h)-\mathscr{B}_{i}(f)(x)\right| \\
& \quad \leq \int_{\mathbb{R}}\left|K_{i}(x+h-y)-K_{i}(x-y)\right||f(y)| d y \\
& \quad \leq f(0) \int_{\mathbb{R}}\left|K_{i}(z+h)-K_{i}(z)\right| d z \longrightarrow 0,
\end{aligned}
$$

as $h \rightarrow 0$ (uniformly in $h$ ), meaning that $\mathscr{B}_{i}(f)$ is equicontinuous in $\mathbb{R}$ due to uniformity of the last estimates in $h$. Moreover, the families $\mathscr{M}_{i}$ are equicontinuous in $\mathbb{R}$, since $\mathscr{B}_{i}\left(f_{i}\right)$ are equicontinuous in $\mathbb{R}$ and the uniform estimate for $f(0)$ in (31). On the other hand, for each $x \in \mathbb{R}$, the set

$$
\mathscr{M}_{i}(x)=\left\{w(x): w \in \mathscr{M}_{i}\right\}
$$

has a compact closure in $\mathbb{R}$ since $\mathscr{M}_{i}(x) \subset\left[0, C_{i}(r)\right]$ for any $x \in \mathbb{R}$. Here $C_{i}(r), i=1,2$, are constants which depend only on $r$. From the Arzela-Ascoli Theorem, the families $\mathscr{M}_{i}$ are normal (see Theorem 1.23, chapter VII in [48]). In other words, we have shown that the set $\mathscr{A}\left(\widetilde{\mathscr{K}}_{r}\right)$ is a relative compact subset of $\widetilde{\mathscr{K}}$. 
Lemma 7. Suppose the same hypothesis in Lemma 6. Let

$$
\begin{aligned}
M & :=\sup _{x \in \mathbb{R}} \max [A(x), B(x), C(x), D(x)], \\
m & :=\inf _{x \in[0,1]} \min [A(x), B(x), C(x), D(x)]>0,
\end{aligned}
$$

and let $R, r$ be constants such that $0<r<1 /(1+\sqrt{2 M})<R<$ 1 , and let $R$ be sufficiently close to 1 .

\section{Then}

(a) $U \neq t \mathscr{A}(U)$ for each $U \in \widetilde{\mathscr{K}} \cap \partial \mathscr{B}_{r}(0)$, and $t \in[0,1]$,

(b) $U-\mathscr{A}(U) \neq a V$ for each $U \in \widetilde{\mathscr{K}} \cap \partial \mathscr{B}_{R}(0)$, and $a \geq 0$, where $V$ is the constant function given by $V=(1,1)^{t}$.

Proof. (a) Suppose that there exist $U=(u, v)^{t} \in \widetilde{\mathscr{K}} \cap \partial \mathscr{B}_{r}(0)$ and $t \in[0,1]$ such that $\widetilde{d}(U, 0)=r$ and

$$
U=t \mathscr{A}(U) \text {. }
$$

Clearly, if $t=0$, we have that $U=0 \notin \widetilde{\mathscr{K}} \cap \partial \mathscr{B}_{r}(0)$ which is a contradiction. Suppose that $t \in(0,1]$. Then we have that $A u^{3}+B v^{2} u$ and $C v^{3}+D u^{2} v$ belong to the cone $\mathscr{K}$ and therefore

$$
\begin{aligned}
& \frac{1}{t} u(0) \leq \int_{\mathbb{R}} K_{1}(-y)\left(A u(y)^{3}+B v(y)^{2} u(y)\right) d y \\
& \leq\left(A(0) u(0)^{3}+B(0) v(0)^{2} u(0)\right) \int_{\mathbb{R}} K_{1}(y) d y, \\
& \frac{1}{t} v(0) \leq \int_{\mathbb{R}} K_{2}(-y)\left(C v(y)^{3}+D u(y)^{2} v(y)\right) d y \\
& \leq\left(C(0) v(0)^{3}+D(0) u(0)^{2} v(0)\right) \int_{\mathbb{R}} K_{2}(y) d y .
\end{aligned}
$$

Thus

$$
\begin{aligned}
& \frac{1}{t} u(0) \leq A(0) u(0)^{3}+B(0) v(0)^{2} u(0), \\
& \frac{1}{t} v(0) \leq C(0) v(0)^{3}+D(0) u(0)^{2} v(0)
\end{aligned}
$$

Since $(u, v)^{t} \in \widetilde{\mathscr{K}} \cap \partial \mathscr{B}_{r}(0)$,

$$
\begin{aligned}
\frac{1}{t} u(0) & \leq A(0)\left(\frac{r}{1-r}\right)^{3}+B(0)\left(\frac{r}{1-r}\right)^{3} \\
& \leq 2 M\left(\frac{r}{1-r}\right)^{3}, \\
\frac{1}{t} v(0) & \leq C(0)\left(\frac{1}{1-r}\right)^{3}+D(0)\left(\frac{r}{1-r}\right)^{3} \\
& \leq 2 M\left(\frac{r}{1-r}\right)^{3} .
\end{aligned}
$$

Due to $\max \{u(0), v(0)\}=r /(1-r)$,

$$
\begin{aligned}
\frac{1}{t}\left(\frac{r}{1-r}\right) & \leq 2 M\left(\frac{r}{1-r}\right)^{3}, \\
\left(\frac{1-r}{r}\right)^{2} & \leq 2 M t .
\end{aligned}
$$

Since $0<t \leq 1$, thus we arrive at

$$
r \geq \frac{1}{1+\sqrt{2 M t}} \geq \frac{1}{1+\sqrt{2 M}}
$$

which contradicts the selection of $r$.

(b) Suppose that there are $U=(u, v)^{t} \in \widetilde{\mathscr{K}} \cap \partial \mathscr{B}_{R}(0)$ and $a \geq 0$ such that

$$
U-\mathscr{A}(U)=a V,
$$

where $V=(1,1)^{t}$. Therefore

$$
\begin{aligned}
& u(x)=a+K_{1} *\left(A u^{3}+B v^{2} u\right), \\
& v(x)=a+K_{2} *\left(C v^{3}+D u^{2} v\right) .
\end{aligned}
$$

As a consequence,

$$
\begin{aligned}
& a+\int_{0}^{1} K_{1} *\left(A u^{3}+B v^{2} u\right) d x=\int_{0}^{1} u(x) d x \\
& \quad \leq\left(\int_{0}^{1} u(x)^{3}\right)^{1 / 3}, \\
& a+\int_{0}^{1} K_{2} *\left(C v^{3}+D u^{2} v\right) d x=\int_{0}^{1} v(x) d x \\
& \quad \leq\left(\int_{0}^{1} v(x)^{3}\right)^{1 / 3} .
\end{aligned}
$$

Let us define, for $i=1,2$,

$$
\kappa_{i}=\int_{0}^{2} K_{i}(z) d z .
$$

Therefore

$$
\begin{aligned}
& \int_{0}^{1} K_{1} *\left(A u^{3}+B v^{2} u\right) d x=\frac{1}{2} \int_{-1}^{1} \int_{\mathbb{R}} K_{1}(x-y) \\
& \cdot\left(A u(y)^{3}+B v(y)^{2} u(y)\right) \geq \frac{1}{2} \iint_{-1}^{1}\left(K_{1}(x-y)\right. \\
& \left.\cdot A u(y)^{3}+K_{1}(x-y) B v(y)^{2} u(y)\right) d x d y \\
& \quad=\frac{1}{2} \int_{-1}^{1} \int_{-1-y}^{1-y}\left(K_{1}(z) A u(y)^{3}\right. \\
& \left.+K_{1}(z) B v(y)^{2} u(y)\right) d z d y=\frac{1}{2} \\
& \cdot \int_{-1}^{1} \int_{-1+y}^{1+y}\left(K_{1}(z) A u(y)^{3}\right. \\
& \left.+K_{1}(z) B v(y)^{2} u(y)\right) d z d y \\
& \geq \kappa_{1} m \int_{0}^{1} u(y)^{3} d y+\kappa_{1} m \int_{0}^{1} v(y)^{2} u(y) d y .
\end{aligned}
$$

Analogously, we obtain that

$$
\begin{aligned}
& \int_{0}^{1} K_{2} *\left(C v^{3}+D u^{2} v\right) d x \\
& \quad \geq \kappa_{2} m \int_{0}^{1} v(y)^{3} d y+\kappa_{2} m \int_{0}^{1} u(y)^{2} v(y) d y .
\end{aligned}
$$


Substituting these the inequalities above into (79), it follows that

$$
\begin{aligned}
a+ & \kappa_{1} m \int_{0}^{1} u(y)^{3} d y+\kappa_{1} m \int_{0}^{1} v(y)^{2} u(y) d y \\
& \leq\left(\int_{0}^{1} u(y)^{3}\right)^{1 / 3}, \\
a & +\kappa_{2} m \int_{0}^{1} v(y)^{3} d y+\kappa_{2} m \int_{0}^{1} u(y)^{2} v(y) d y \\
& \leq\left(\int_{0}^{1} v(y)^{3}\right)^{1 / 3} .
\end{aligned}
$$

We conclude that $\int_{0}^{1} u(y)^{3} d y$ and $\int_{0}^{1} v(y)^{3} d y$ are bounded by a constant for all $(u, v) \in \widetilde{\mathscr{K}} \cap \partial \mathscr{B}_{R}(0)$, satisfying (77). In consequence, $\int_{0}^{1} v(y)^{2} u(y) d y$ and $\int_{0}^{1} u(y)^{2} v(y) d y$ are also bounded by a constant for all $(u, v) \in \widetilde{\mathscr{K}} \cap \partial \mathscr{B}_{R}(0)$, satisfying (77).

On the other hand, from (78)

$$
\begin{aligned}
0 \leq & u(x) \\
= & a+\int_{\mathbb{R}} K_{1}(x-y) A u(y)^{3} d y \\
& +\int_{\mathbb{R}} K_{1}(x-y) B v(y)^{2} u(y) d y \\
= & a+\sum_{j=-\infty}^{\infty} \int_{2 j-1}^{2 j+1} K_{1}(x-y) A u(y)^{3} d y \\
& +\sum_{j=-\infty}^{\infty} \int_{2 j-1}^{2 j+1} K_{1}(x-y) B v(y)^{2} u(y) d y, \\
0 \leq & v(x) \\
= & a+\int_{\mathbb{R}} K_{2}(x-y) C v(y)^{3} d y \\
& +\int_{\mathbb{R}} K_{2}(x-y) D u(y)^{2} v(y) d y \\
= & a+\sum_{j=-\infty}^{\infty} \int_{2 j-1}^{2 j+1} K_{2}(x-y) C v(y)^{3} d y \\
& +\sum_{j=-\infty}^{\infty} \int_{2 j-1}^{2 j+1} K_{2}(x-y) D u(y)^{2} v(y) d y .
\end{aligned}
$$

In order to bound the right-hand side of the inequalities above, observe that

$$
\begin{aligned}
& \sum_{j=-\infty}^{\infty} \int_{2 j-1}^{2 j+1} K_{1}(x-y) A u(y)^{3} d y=\int_{-1}^{1}\left[K_{1}(x-y)\right. \\
& \cdot A u(y)^{3} d y \\
& \quad+\sum_{j=1}^{\infty}\left(K_{1}(x-y-2 j)+K_{1}(x-y+2 j)\right)
\end{aligned}
$$

$$
\begin{aligned}
& \left.\cdot A u(y+2 j)^{3}\right] d y \leq M \int_{-1}^{1}\left[K_{1}(x-y)\right. \\
& \left.+\sum_{j=1}^{\infty}\left(K_{1}(x-y-2 j)+K_{1}(x-y+2 j)\right)\right] \\
& \cdot u(y)^{3} d y=M \int_{-1}^{1} \sum_{j=-\infty}^{\infty} K_{1}(x-y+2 j) \\
& \cdot u(y)^{3} d y \leq M \max _{-1 \leq z \leq 1} \sum_{j=-\infty}^{\infty} K_{1}(z+2 j) \\
& \cdot \int_{-1}^{1} u(y)^{3} d y \leq 2 M \max _{-1 \leq z \leq 1} \sum_{j=-\infty}^{\infty} K_{1}(z+2 j) \\
& \cdot \int_{0}^{1} u(y)^{3} d y .
\end{aligned}
$$

Now, since $K_{1} \in L^{1}(\mathbb{R}) \cap L^{\infty}(\mathbb{R})$ is monotone decreasing on $[0, \infty)$, the function defined by

$$
\widetilde{K}_{1}(x):=\sum_{j=-\infty}^{\infty} K_{1}(x+2 j)
$$

is periodic and monotone decreasing on $[0,1]$ and belongs to $L^{1}(0,1)$. Therefore,

$$
\max _{-1 \leq z \leq 1} \widetilde{K}_{1}(z)=\sum_{j=-\infty}^{\infty} K_{1}(2 j),
$$

and thus

$$
\begin{aligned}
& \sum_{j=-\infty}^{\infty} \int_{2 j-1}^{2 j+1} K_{1}(x-y) A u(y)^{3} d y \\
& \quad \leq 2 M \sum_{j=-\infty}^{\infty} K_{1}(2 j) \int_{0}^{1} u(y)^{3} d y .
\end{aligned}
$$

Analogously,

$$
\begin{aligned}
& \sum_{j=-\infty}^{\infty} \int_{2 j-1}^{2 j+1} K_{1}(x-y) B v(y)^{2} u(y) d y \\
& \leq 2 M \sum_{j=-\infty}^{\infty} K_{1}(2 j) \int_{0}^{1} v(y)^{2} u(y) d y, \\
& \sum_{j=-\infty}^{\infty} \int_{2 j-1}^{2 j+1} K_{2}(x-y) C v(y)^{3} d y \\
& \leq 2 M \sum_{j=-\infty}^{\infty} K_{2}(2 j) \int_{0}^{1} v(y)^{3} d y, \\
& \sum_{j=-\infty}^{\infty} \int_{2 j-1}^{2 j+1} K_{2}(x-y) D u(y)^{2} v(y) d y \\
& \leq 2 M \sum_{j=-\infty}^{\infty} K_{2}(2 j) \int_{0}^{1} u(y)^{2} v(y) d y,
\end{aligned}
$$


where $\widetilde{K}_{2}$ is the periodic function defined by

$$
\widetilde{K}_{2}(x):=\sum_{j=-\infty}^{\infty} K_{2}(x+2 j),
$$

with

$$
\max _{-1 \leq z \leq 1} \widetilde{K}_{2}(z)=\sum_{j=-\infty}^{\infty} K_{2}(2 j) .
$$

Introducing these results into (84), we arrive at

$$
\begin{aligned}
0 \leq & u(x) \\
\leq & a+2 M \sum_{j=-\infty}^{\infty} K_{1}(2 j) \int_{0}^{1} u(y)^{3} d y \\
& +2 M \sum_{j=-\infty}^{\infty} K_{1}(2 j) \int_{0}^{1} v(y)^{2} u(y) d y, \\
0 \leq & v(x) \\
\leq & a+2 M \sum_{j=-\infty}^{\infty} K_{2}(2 j) \int_{0}^{1} v(y)^{3} d y \\
& +2 M \sum_{j=-\infty}^{\infty} K_{2}(2 j) \int_{0}^{1} u(y)^{2} v(y) d y .
\end{aligned}
$$

From (83), we get that $a \leq\left(\int_{0}^{1} u(y)^{3} d y\right)^{1 / 3}$ and $a \leq$ $\left(\int_{0}^{1} v(y)^{3} d y\right)^{1 / 3}$. Therefore

$$
\begin{aligned}
0 \leq & u(x) \\
\leq & \left(\int_{0}^{1} u(y) d y\right)^{1 / 3}+2 M \sum_{j=-\infty}^{\infty} K_{1}(2 j) \int_{0}^{1} u(y)^{3} d y \\
& +2 M \sum_{j=-\infty}^{\infty} K_{1}(2 j) \int_{0}^{1} v(y)^{2} u(y) d y:=\kappa, \\
0 \leq & v(x) \\
\leq & \left(\int_{0}^{1} v(y) d y\right)^{1 / 3}+2 M \sum_{j=-\infty}^{\infty} K_{2}(2 j) \int_{0}^{1} v(y)^{3} d y \\
& +2 M \sum_{j=-\infty}^{\infty} K_{2}(2 j) \int_{0}^{1} u(y)^{2} v(y) d y:=\mu .
\end{aligned}
$$

Thus if we select $R$ sufficiently close to 1 , such that

$$
\frac{\max \{\kappa, \mu\}}{1+\max \{\kappa, \mu\}}<R<1,
$$

we get that

$$
\max \{u(0), v(0)\}<\max \{\kappa, \mu\}<\frac{R}{1-R} .
$$

We conclude that

$$
\tilde{d}((u, v), 0)=\frac{\max \{u(0), v(0)\}}{1+\max \{u(0), v(0)\}}<R,
$$

which is a contradiction.
Theorem 8. Suppose the same hypothesis on the coefficients $A, B, C, D, \beta_{1}, \beta_{2}$, and $r$ and $R$ as in Lemma 7. Furthermore, assume that system (18) does not have constant solutions $(\widetilde{u}, \widetilde{v}) \neq(0,0)$. Then the operator $\mathscr{A}$ has a nontrivial (i.e., $\tilde{u}$ or $\widetilde{v}$ is not constant) fixed point in the cone $\widetilde{\mathscr{K}}$. Equivalently, there exists a nontrivial solution of system (18). Moreover, the fixed point index of the operator $\mathscr{A}$ on $\widetilde{\mathscr{K}}_{r}^{R}$ is $i\left(\widetilde{\mathscr{K}}, \mathscr{A}, \widetilde{\mathscr{K}}_{r}^{R}\right)=-1$.

Proof. Observe that $(0,0)$ is a trivial solution of system (18). However $(0,0)$ does not belong to the annular region $\widetilde{\mathscr{K}}_{r}^{R}$. Thus, Lemmas 6 and 7 and Theorem 3 guarantee the existence of a nontrivial fixed point (i.e., $\widetilde{u}$ or $\widetilde{v}$ is not constant) of the operator $\mathscr{A}$ in the annular region $\widetilde{\mathscr{K}}_{r}^{R}=\{U \in \mathscr{K}: r<$ $\tilde{d}(U, 0)<R\} \subset \widetilde{\mathscr{K}}$.

\section{Numerical Results}

As mentioned above, explicit solutions of full system (1) are not known for general coefficients $a(x), b(x), c(x), d(x)$, and $e(x)$. In this section we will describe a numerical strategy to compute approximations of vector solitons $(u, v)$ of this system in the form

$$
\begin{aligned}
& u(\xi, x)=e^{i \alpha \xi} \widetilde{u}(x), \\
& v(\xi, x)=e^{i \alpha \xi} \widetilde{v}(x),
\end{aligned}
$$

when $\delta=0$,

or

$$
\begin{aligned}
& u(\xi, x)=e^{i \alpha_{1} \xi-(i \delta / 2 K) x} \widetilde{u}(x), \\
& v(\xi, x)=e^{i \alpha_{2} \xi+(i \delta / 2 K) x} \widetilde{v}(x), \\
& \text { when } e \equiv 0,
\end{aligned}
$$

where $\widetilde{u}$ and $\widetilde{v}$ are positive, even, and real solutions of the system

$$
\begin{aligned}
& \left(I-\beta_{1} \partial_{x}^{2}\right) \tilde{u}=A(x) \tilde{u}^{3}+B(x) \tilde{v}^{2} \tilde{u} \\
& \left(I-\beta_{2} \partial_{x}^{2}\right) \tilde{v}=C(x) \tilde{v}^{3}+D(x) \tilde{u}^{2} \widetilde{v}
\end{aligned}
$$

The existence of such solutions was already established in the previous section.

To approximate these solutions, following the same collocation-spectral strategy by Pipicano and Muñoz Grajales in [49], let us introduce truncated $2 l$-periodic cosine expansions for $\widetilde{u}$ and $\widetilde{v}$ :

$$
\begin{aligned}
& \widetilde{u}(x) \approx \zeta_{0}+\sum_{n=1}^{N / 2} \widetilde{u}_{n} \cos \left(\frac{n \pi}{l} x\right), \\
& \widetilde{v}(x) \approx \widetilde{u}_{0}+\sum_{n=1}^{N / 2} \widetilde{v}_{n} \cos \left(\frac{n \pi}{l} x\right),
\end{aligned}
$$

where

$$
\begin{aligned}
& \widetilde{u}_{0}=\frac{1}{l} \int_{0}^{l} \widetilde{u}(x) d x=\frac{1}{2 l} \int_{0}^{2 l} \widetilde{u}(x) d x, \\
& \tilde{u}_{n}=\frac{2}{l} \int_{0}^{l} \widetilde{v}(x) \cos \left(\frac{n \pi x}{l}\right) d x
\end{aligned}
$$



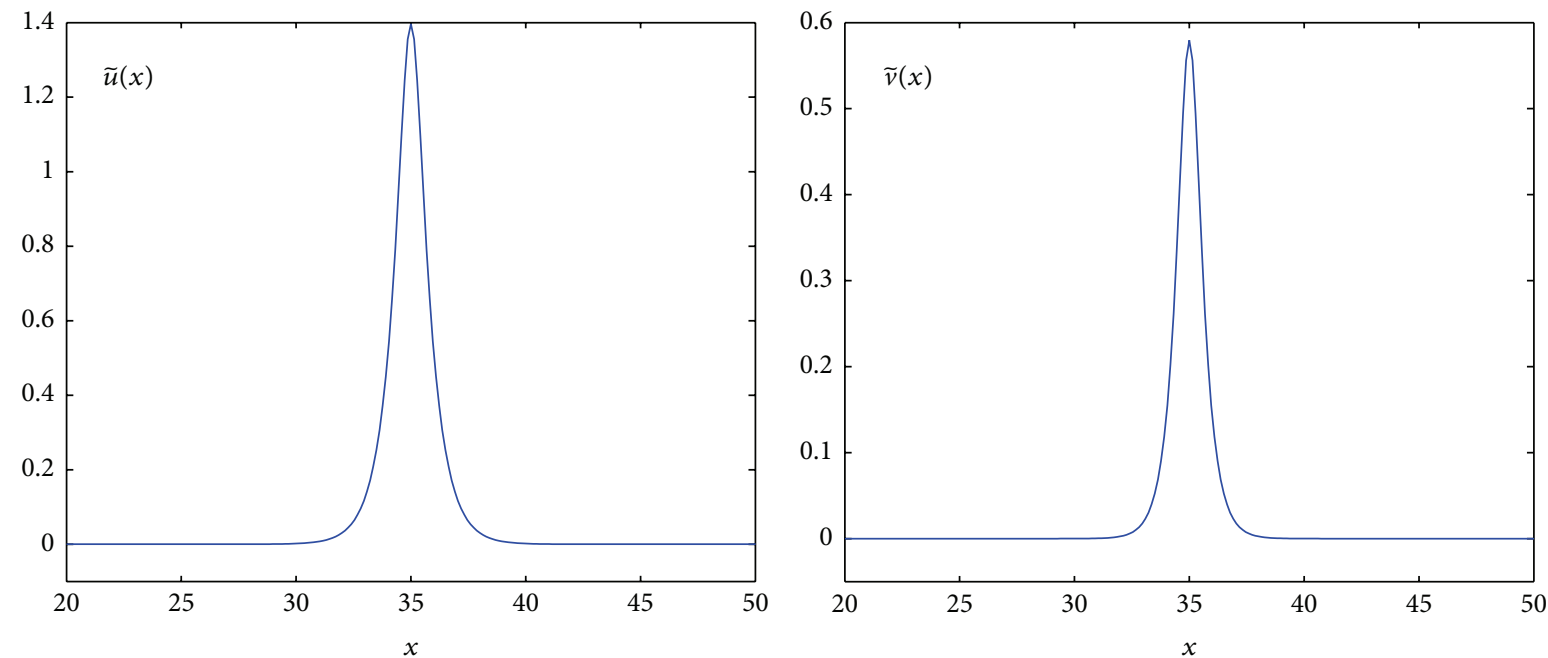

Figure 1: Vector soliton of system (1) obtained after 10 Newton's iterations and $\delta=0, \sigma_{1}=0.5, \sigma_{2}=-0.5, \alpha=1.5$, and $K=0.5$.
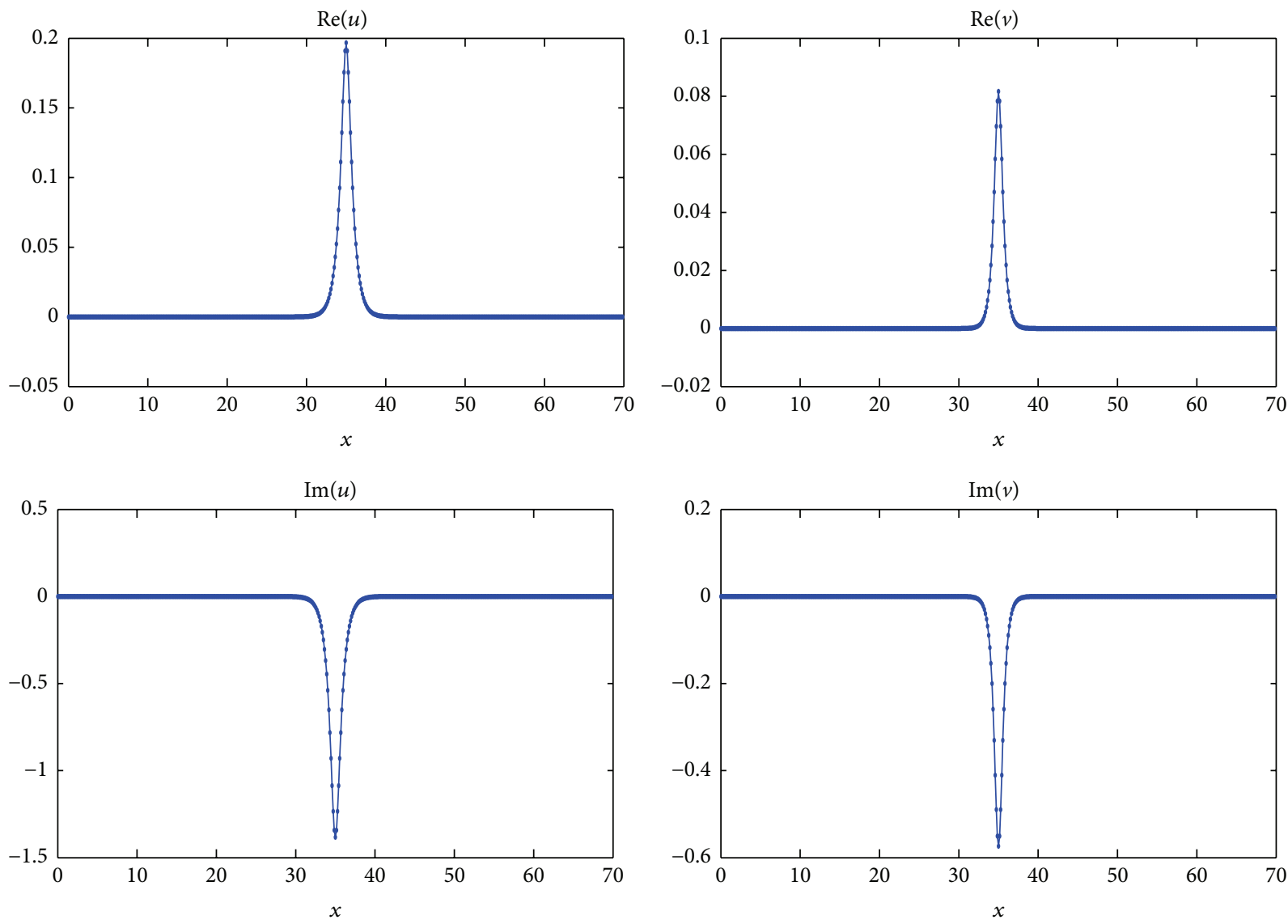

Figure 2: Real and imaginary parts of the vector soliton $(u, v)$ of system (1) at $\xi=20$. Solid line: numerical solution $(u, v)$ of system $(1)$ using the numerical scheme in [37]. Pointed line: expected soliton computed using (108).

$$
=\frac{2}{2 l} \int_{0}^{2 l} \widetilde{u}(x) \cos \left(\frac{n \pi x}{l}\right) d x
$$

and let us introduce analogous expressions for the coefficients $\widetilde{v}_{n}$. This strategy can be used for approximating solutions decaying to zero at infinity, provided that the period $2 l$ is taken large enough.

By substituting expressions (100) into (99), evaluating them at the $N / 2+1$ collocation points

$$
x_{j}=\frac{2 l(j-1)}{N}, \quad j=1, \ldots, \frac{N}{2}+1,
$$



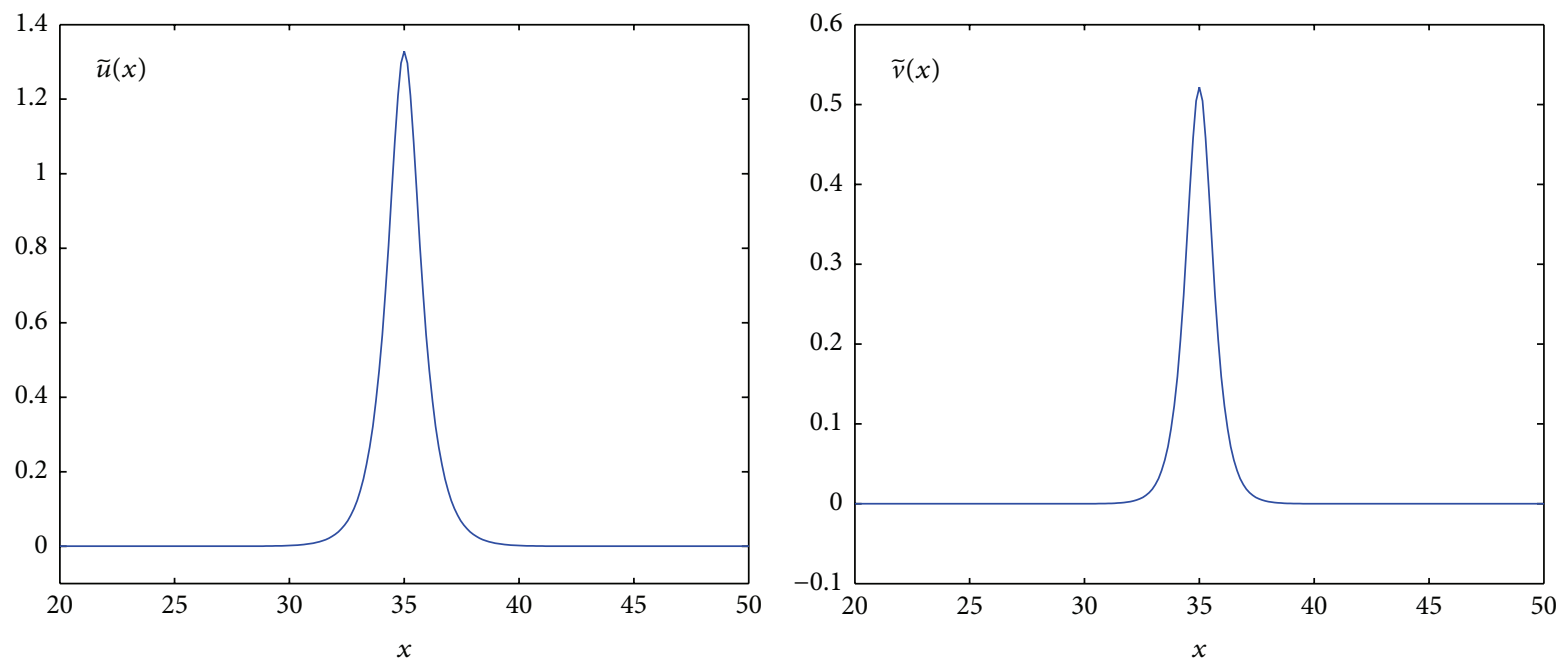

Figure 3: Vector soliton of system (1) obtained after 10 Newton's iterations and $\delta=0, \sigma_{1}=0.5, \sigma_{2}=-0.5, \alpha=1.5$, and $K=0.5$.
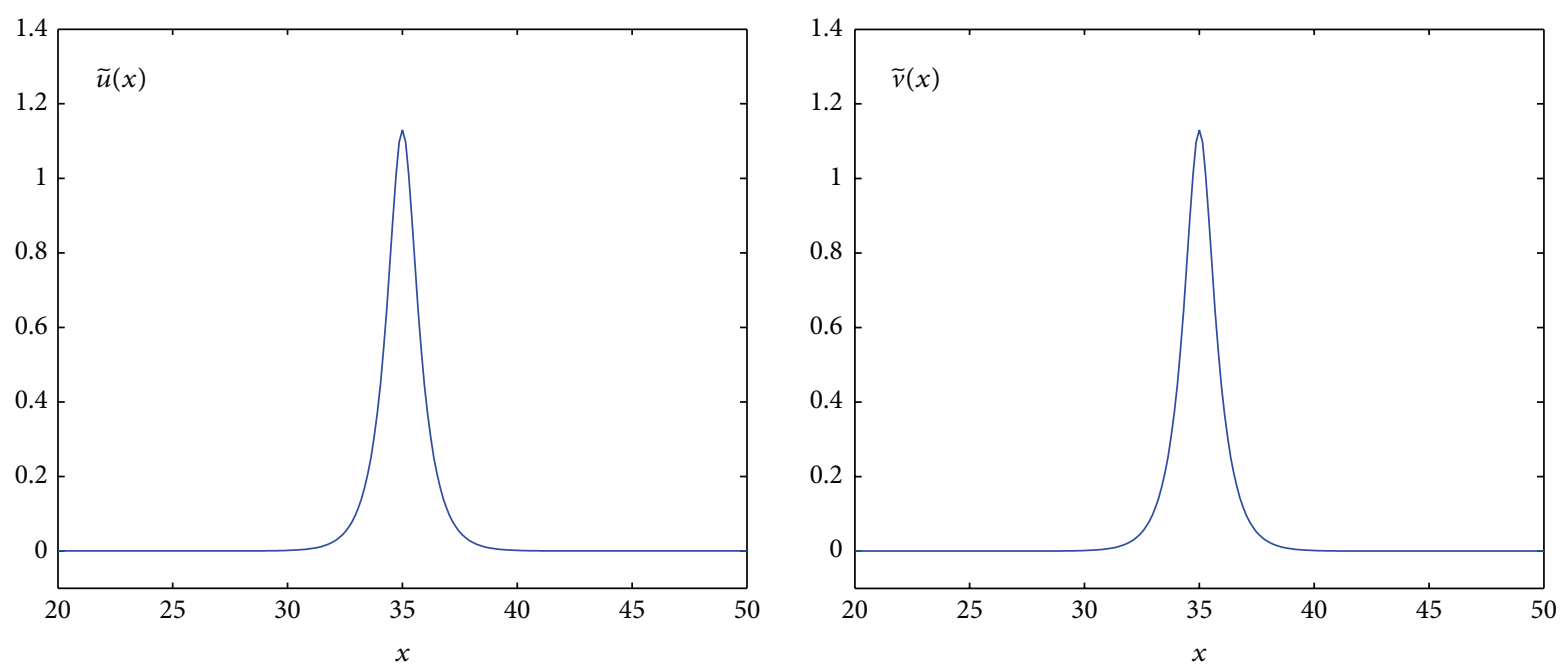

Figure 4: Vector soliton of system (1) obtained after 10 Newton's iterations and $\delta=1, \sigma_{1}=\sigma_{2}=0$, and $\alpha_{1}=\alpha_{2}=1.5$.

we obtain a system of $N+2$ nonlinear equations in the form

$$
F\left(\tilde{u}_{0}, \tilde{u}_{1}, \ldots, \tilde{u}_{N / 2}, \widetilde{v}_{0}, \widetilde{v}_{1}, \ldots, \widetilde{v}_{N / 2}\right)=0,
$$

where $N+2$ coefficients $\widetilde{u}_{n}$ and $\widetilde{v}_{n}$ are the unknowns. Nonlinear system (103) can be solved by Newton's iteration. Computation of the cosine series in (100) and the integrals in (101) is performed using the FFT (Fast Fourier Transform) algorithm. The Jacobian of the vector field $F: \mathbb{R}^{N+2} \rightarrow \mathbb{R}^{N+2}$ is approximated by the second-order accurate formula

$$
\begin{aligned}
J_{i, j} F(x) \approx \frac{F_{i}\left(x+h e_{j}\right)-F_{i}\left(x-h e_{j}\right)}{2 h} & \\
j=1, \ldots, N+2, &
\end{aligned}
$$

where $e_{j}=(0, \ldots, 1, \ldots, 0)$ and $h=0.01$. We stop the iteration procedure when the relative error between two successive approximations and the value of the vector field $F$ are smaller than $10^{-12}$.
5.1. Description of the Numerical Experiments. In all numerical experiments the number of FFT points is $N=2^{9}$ and $l=35$. Thus the computational domain of the variable $x$ is $[0,70]$.

Experiment Set 1 (vanishing inverse group velocity). We will first consider the regime where $\delta=0, \sigma_{1}=0.5, \sigma_{2}=-0.5$, $\alpha=1.5$, and $K=0.5$. Therefore $\beta_{1}=0.5$ and $\beta_{2}=0.25$. The coefficients in system (1) are taken as

$$
\begin{aligned}
a(x) & =b(x)=c(x)=d(x)=e(x) \\
& =\operatorname{sech}^{p}\left(x-x_{0}\right)
\end{aligned}
$$

with $p=3$ and $x_{0}=35$. Note that the parameter $x_{0}$ regulates the position of the irregular section of the coefficients $a(x)$, $b(x), c(x), d(x)$, and $e(x)$. The initial values for the Newton's iteration are

$$
\widetilde{u}(x)=\widetilde{v}(x)=\operatorname{sech}\left(x-x_{0}\right) .
$$



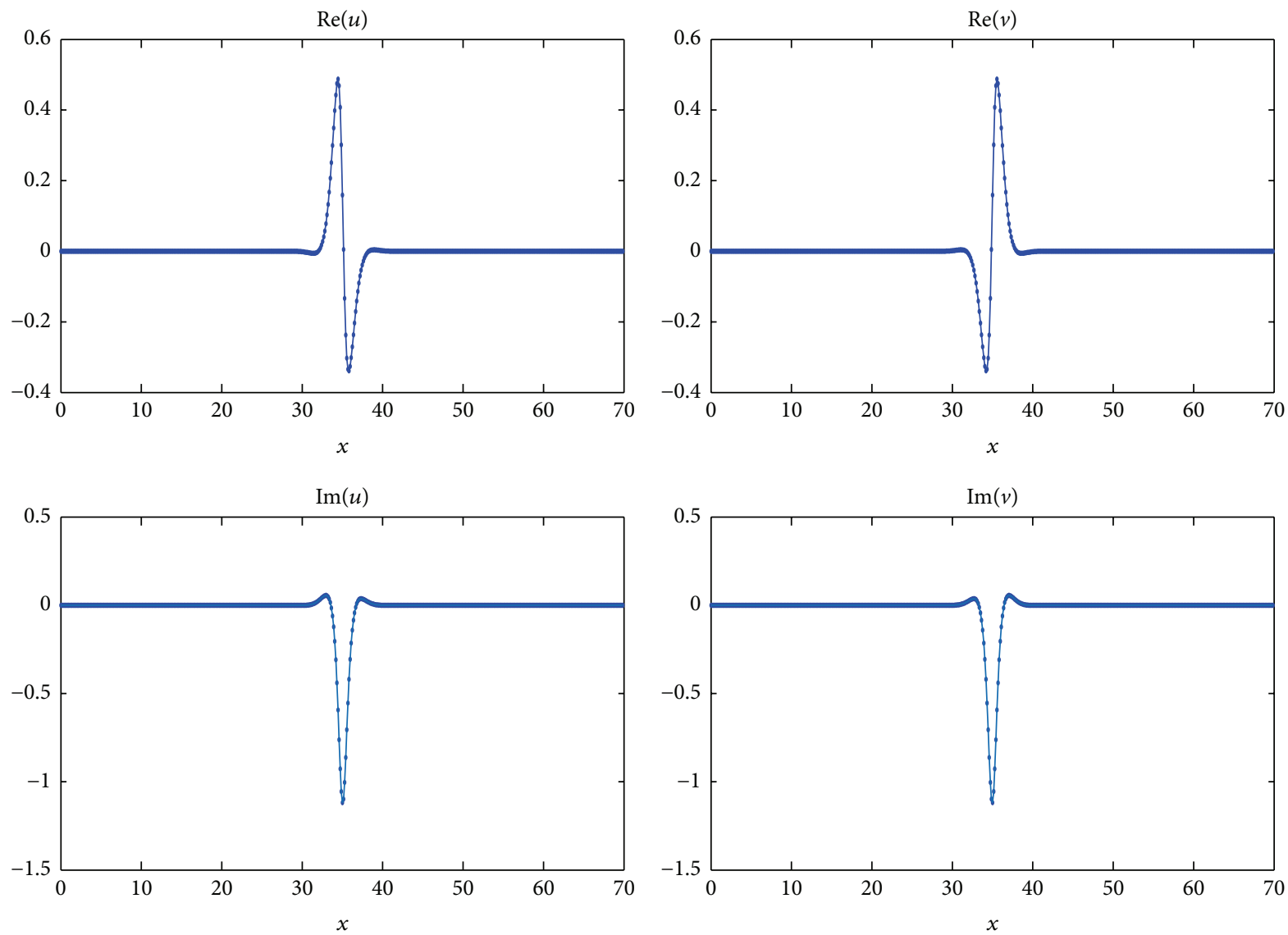

FIGURE 5: Real and imaginary parts of the vector soliton $(u, v)$ of system $(1)$ at $\xi=20$. Solid line: numerical solution $(u, v)$ of system $(1)$ using the numerical scheme in [37]. Pointed line: expected soliton computed using (111).

The resulting approximations of $(\widetilde{u}, \widetilde{v})$ after 10 iterations are presented in Figure 1. To verify that we have computed really a vector soliton of system (1), we run the numerical solver to approximate the solutions to system (1), introduced by Muñoz Grajales and Quiceno in [37], with stepsize $\Delta \xi=10^{-3}$, $N=2^{9}$ FFT points, using as initial data

$$
\begin{aligned}
& u(0, x)=\widetilde{u}(x), \\
& v(0, x)=\widetilde{v}(x) .
\end{aligned}
$$

The result of this computer simulation at $\xi=20$ is presented in Figure 2, superimposed with the expected position of the vector soliton given by

$$
\begin{aligned}
& u(\xi, x)=e^{i \alpha \xi} \widetilde{u}(x), \\
& v(\xi, x)=e^{i \alpha \xi} \widetilde{v}(x) .
\end{aligned}
$$

We observe a good accordance between the profiles of the components $u$ and $v$, corroborating that, in fact, we have an approximation of a vector soliton to system (1).
We repeat the numerical experiments above with the same model and numerical parameters, but now for Gaussian-type coefficients,

$$
\begin{aligned}
a(x) & =b(x)=c(x)=d(x)=e(x) \\
& =\exp \left(-0.5\left(x-x_{0}\right)^{2}\right) .
\end{aligned}
$$

The results are presented in Figure 3.

Experiment Set 2 (nonzero inverse group velocity). We now set $\delta=1, \sigma_{1}=\sigma_{2}=0$, and $\alpha_{1}=\alpha_{2}=1.5$. Thus $\beta_{1}=\beta_{2}=$ 0.5 . We point out that, in this case, $\delta \neq 0$, in contrast to the simulations above. The model's coefficients in system (18) are taken as

$$
\begin{aligned}
& a(x)=b(x)=c(x)=d(x)=\operatorname{sech}^{p}\left(x-x_{0}\right), \\
& e(x) \equiv 0 .
\end{aligned}
$$

The resulting approximation to the pair $(\widetilde{u}, \widetilde{v})$ obtained from Newton's procedure is presented in Figure 4 and the verification of the characteristics of a soliton solution is 

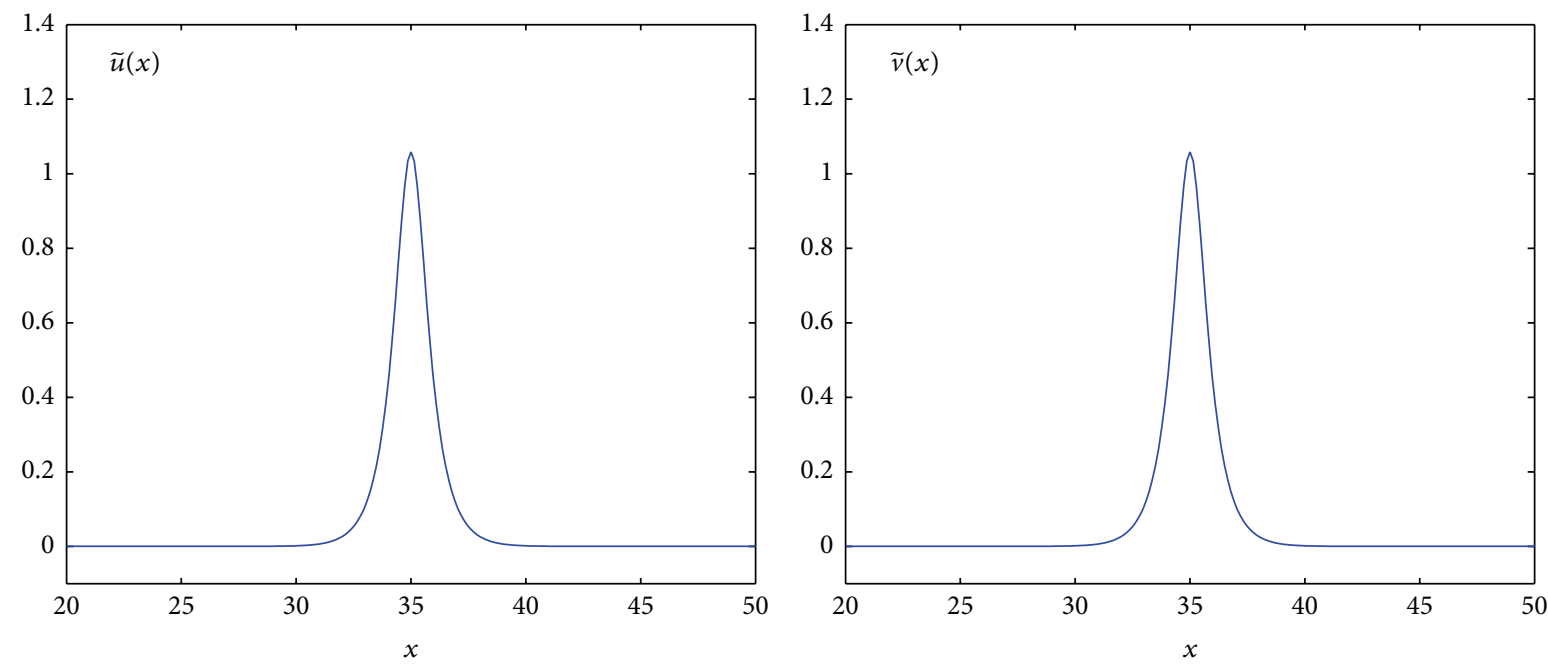

Figure 6: Vector soliton of system (1) obtained after 10 Newton's iterations and $\delta=1, \sigma_{1}=\sigma_{2}=0$, and $\alpha_{1}=\alpha_{2}=1.5$.
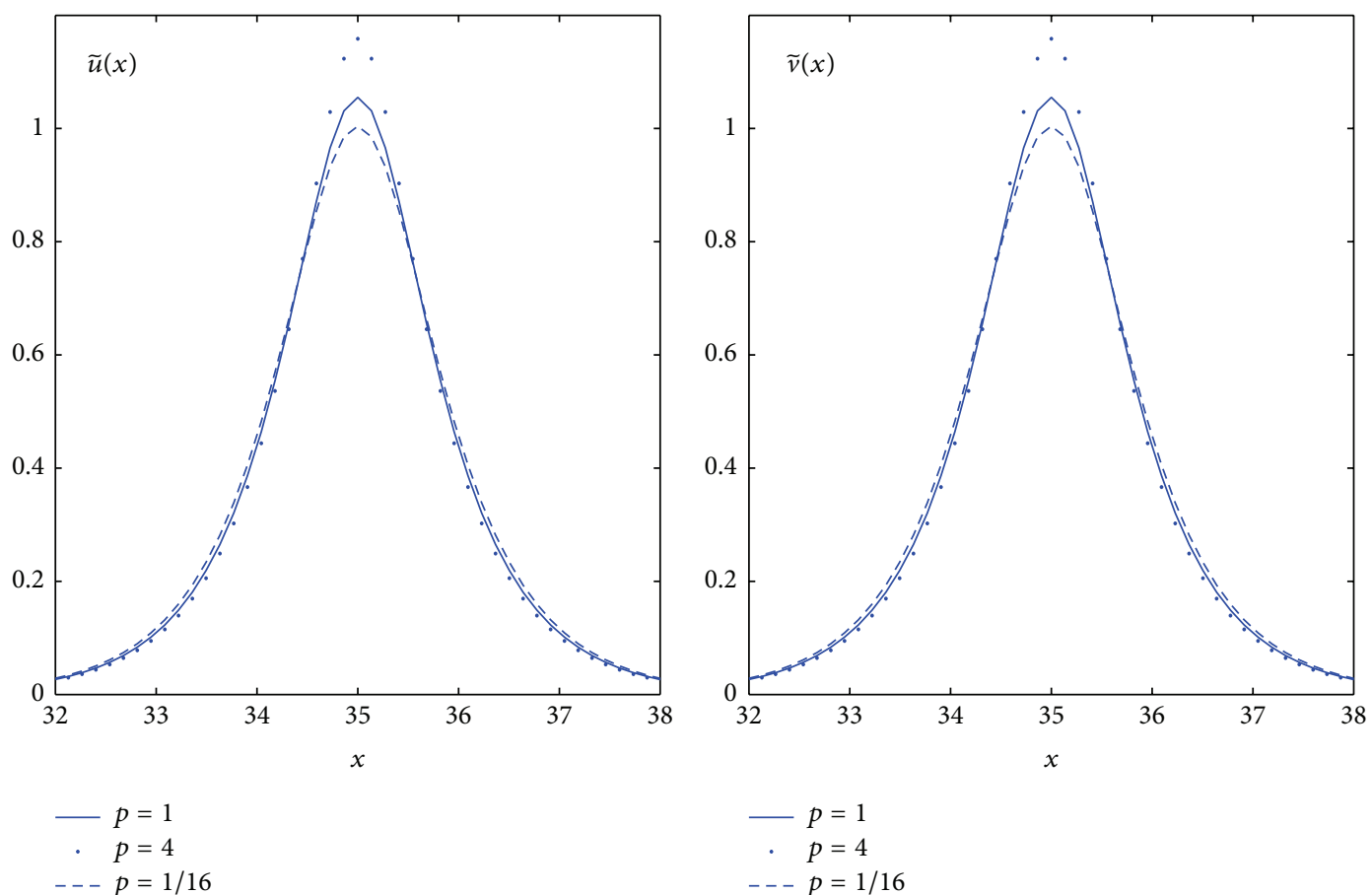

FIGURE 7: Effect of the model's coefficients $a, b, c, d$ on the shape of the soliton solutions. Here $e \equiv 0, \delta=1, \alpha_{1}=\alpha_{2}=1.5, \sigma_{1}=\sigma_{2}=0$, $K=0.5, x_{0}=35$, and $a(x)=b(x)=c(x)=d(x)=\operatorname{sech}^{p}\left(x-x_{0}\right)$, with $p=1,4,1 / 16$.

displayed in Figure 5. Note that, within this parameter regime, the expected soliton solution is given by

$$
\begin{aligned}
& u(\xi, x)=e^{i \alpha_{1} \xi-(i \delta / 2 K) x} \widetilde{u}(x), \\
& v(\xi, x)=e^{i \alpha_{2} \xi+(i \delta / 2 K) x} \widetilde{v}(x) .
\end{aligned}
$$

We repeat the previous numerical experiment with the same model and numerical parameters but instead use the coefficients

$$
\begin{aligned}
a(x) & =b(x)=c(x)=d(x)=e(x) \\
& =\exp \left(-0.5\left(x-x_{0}\right)^{2}\right) .
\end{aligned}
$$

The results are presented in Figure 6. In all cases, we performed a verification of the approximate soliton solution computed using the numerical scheme developed in [37] for solving coupled system (1). The error observed at $\xi=20$ was around $10^{-3}$.

Experiment Set 3 (effect of inhomogeneous coefficients). The next step is to analyze the effect of the model's coefficients on the geometry of the computed vector solitons of (18). In the simulations presented in Figure 7, we set $e \equiv 0, \delta=1$, $\alpha_{1}=\alpha_{2}=1.5, \sigma_{1}=\sigma_{2}=0, K=0.5, x_{0}=35$, and $a(x)=$ $b(x)=c(x)=d(x)=\operatorname{sech}^{p}\left(x-x_{0}\right)$. The other numerical 

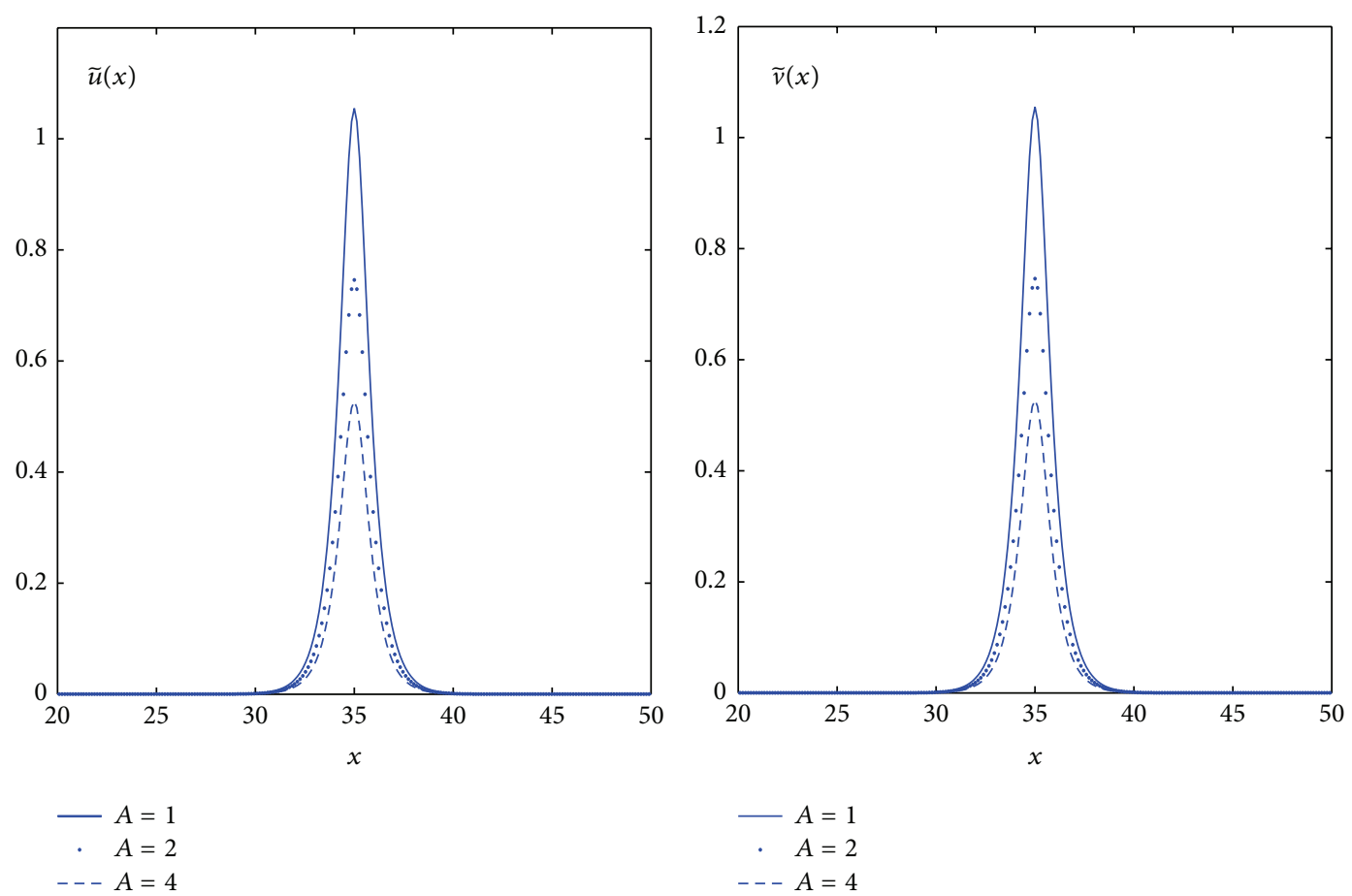

FIGURE 8: Effect of the model's coefficients on the shape of the soliton solutions. Here $e \equiv 0, \delta=1, \alpha_{1}=\alpha_{2}=1.5, \sigma_{1}=\sigma_{2}=0, K=0.5$, $x_{0}=35$, and $a(x)=b(x)=c(x)=d(x)=A \operatorname{sech}\left(x-x_{0}\right)$, with $A=1$ (solid line), $A=2$ (pointed line), and $A=4$ (dashed line).
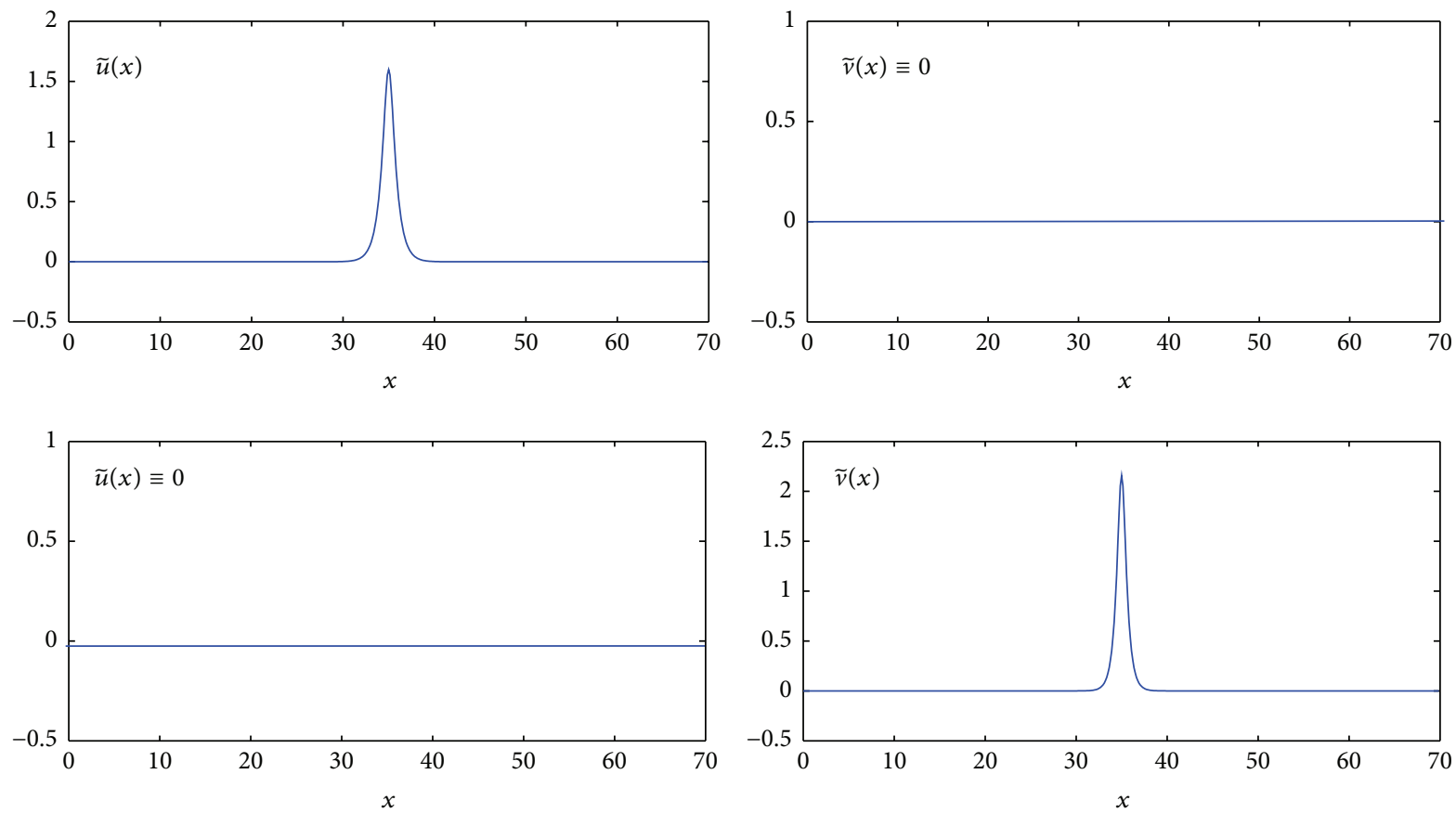

FigurE 9: Single-mode solitons for system (18).

parameters are left unchanged. Observe that the amplitude of the approximate solution decreases when $p \rightarrow 0$.

On the other hand, in Figure 8, we let $a(x)=b(x)=$ $c(x)=d(x)=A \operatorname{sech}\left(x-x_{0}\right)$ and compute the solution of system (18) for different values of the amplitude $A$. Other parameters are the same as in the previous experiment.
However, for $A=2,4$, we have to start Newton's iteration with the following data:

$$
\begin{aligned}
& \widetilde{u}=0.5 \operatorname{sech}\left(x-x_{0}\right), \\
& \widetilde{v}=0.5 \operatorname{sech}\left(x-x_{0}\right),
\end{aligned}
$$



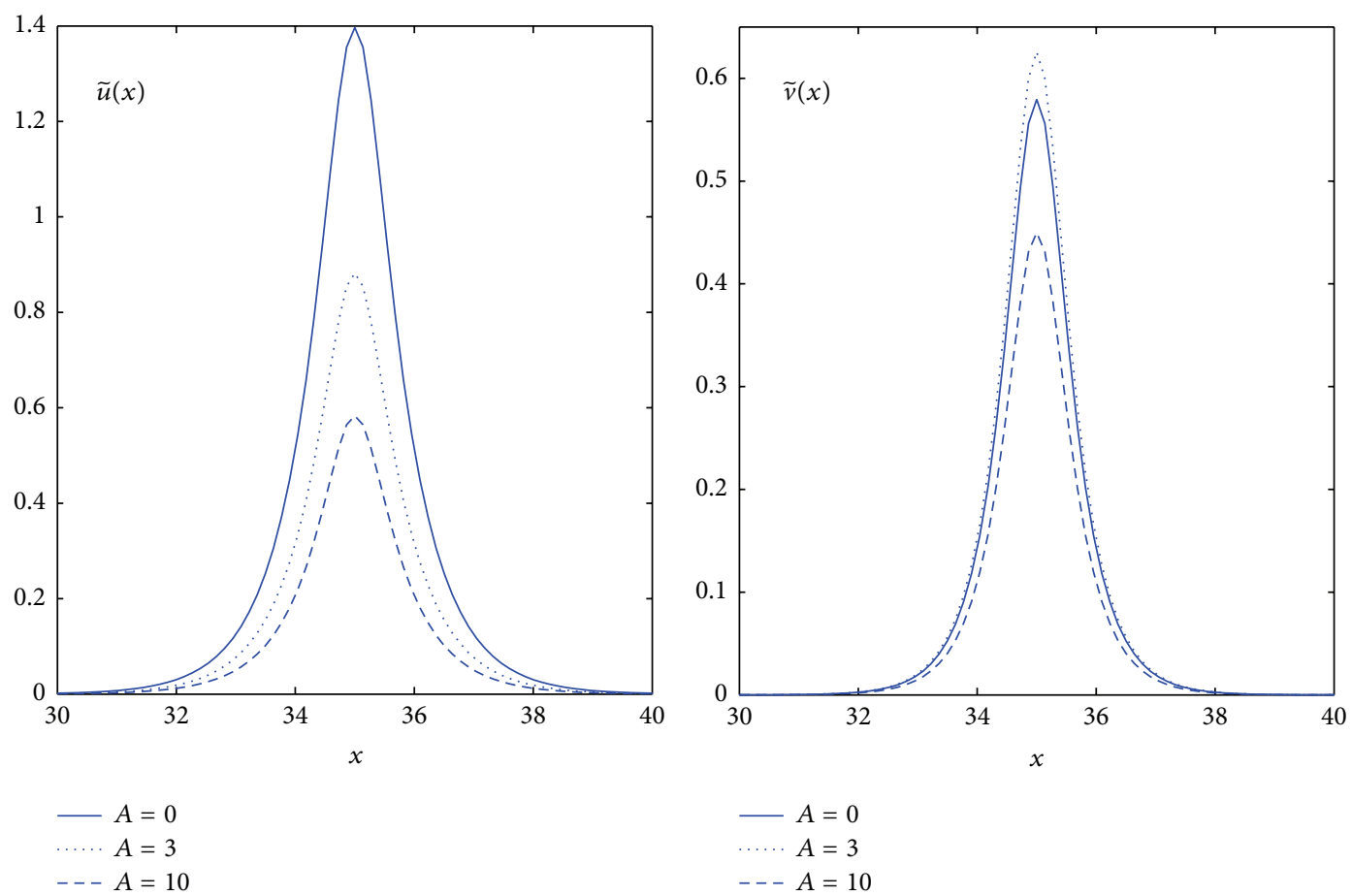

FIGURE 10: Effect of the inhomogeneous coefficient $e(x)$ on the shape of the vector soliton. Here $\delta=0, \alpha=1.5, \sigma_{1}=0.5, \sigma_{2}=-0.5, K=0.5$, $x_{0}=35, a(x)=c(x)=\operatorname{sech}^{p}\left(x-x_{0}\right), b(x)=d(x)=2 \operatorname{sech}^{p}\left(x-x_{0}\right), e(x)=A \operatorname{sech}^{p}\left(x-x_{0}\right)$, and $p=3$. Solid line: $A=0$; that is, $e \equiv 0$. Pointed line: $A=3$. Dashed line: $A=10$.

$|u|$

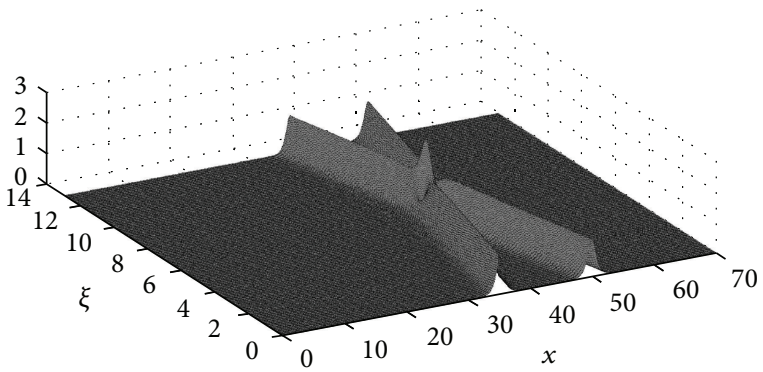

FIGURE 11: Collision of coupled-mode solitons with $\phi_{1}=\phi_{2}=0$.

in order to achieve convergence. From these results, we have numerical evidence on the fact that the amplitude of the solutions of system (18) decreases as the amplitude of the coefficients $a(x), b(x), c(x)$, and $d(x)$ increases.

Experiment Set 4 (single-mode solitons). When $v \equiv 0$ or $u \equiv 0$, the cross-modulation terms in system (1) are zero, obtaining that $u$ or $v$, respectively, satisfy a scalar Schrödinger equation. The single-mode solitons computed are displayed in Figure 9 for the same model and numerical parameters as in the Experiment Set 1.

Experiment Set 5 (influence of the inhomogeneous coefficient $e(x))$. In Figure 10, we illustrate the effect of the variable coefficient $e(x)$ on the shape of the vector solitons of system

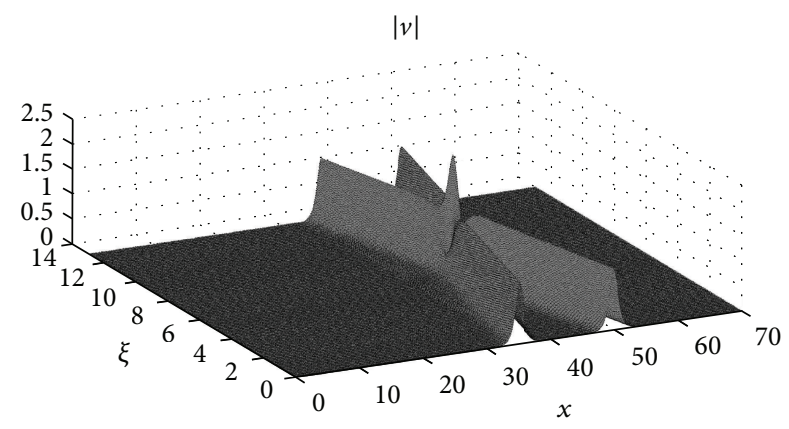

FIGURE 12: Collision of coupled-mode solitons with $\phi_{1}=\phi_{2}=0$.

(1). The model's parameters are $K=0.5, \alpha=1.5, \sigma_{1}=0.5$, $\sigma_{2}=-0.5, \delta=0$, and

$$
\begin{aligned}
& a(x)=c(x)=\operatorname{sech}^{p}\left(x-x_{0}\right), \\
& b(x)=d(x)=2 \operatorname{sech}^{p}\left(x-x_{0}\right), \\
& e(x)=A \operatorname{sech}^{p}\left(x-x_{0}\right),
\end{aligned}
$$

with $p=3$ and $x_{0}=35$. The initial values for the Newton iteration in this case are taken as

$$
\widetilde{u}(x)=\widetilde{v}(x)=\operatorname{sech}^{p}\left(x-x_{0}\right) .
$$

In solid line, we display the vector soliton computed using $A=0$, that is, $e \equiv 0$, superimposed with the profiles obtained for nonzero values of this parameter with $A=3$ and 


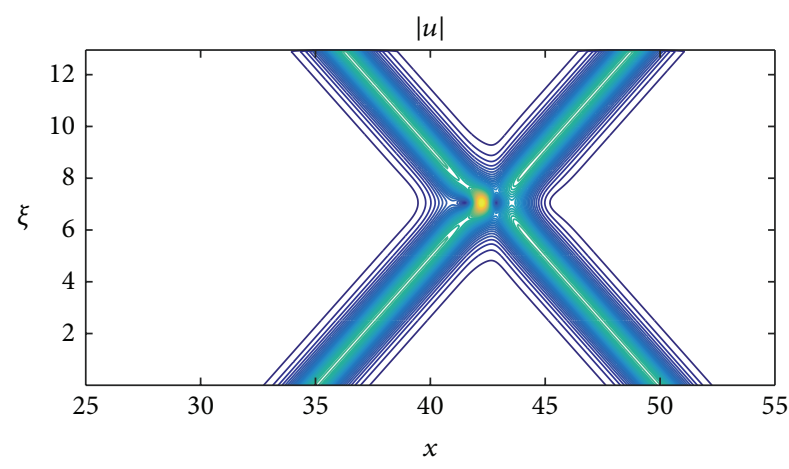

FIGURE 13: Contour plot of the collision of solitons in Figure 11.

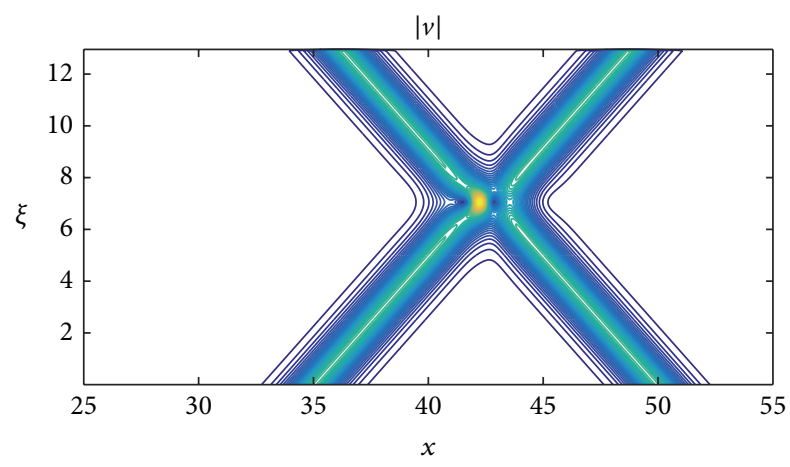

FIGURE 14: Contour plot of the collision of solitons in Figure 12.

$A=10$. Observe that the intensity of the extrainhomogeneous nonlinearities in system (1), produced by the coefficient $e(x)$, affect significantly the amplitude of the vector soliton obtained.

Experiment Set 6 (interaction of vector solitons). We are interested in illustrating the collision of coupled-mode waves in the regime where $\delta=0$ and all model's coefficients are constants. By using the Galilean invariance of system (1) (see Theorem 4), we can write the vector solitons in a more general form, by considering a nonzero translational velocity. Thus, we run again the numerical solver in [37] with an initial profile consisting of two well-separated solitons:

$$
\begin{aligned}
u(0, x)= & \widetilde{u}(x) e^{\left(i V_{1} / 2 K\right) x} \\
& +\widetilde{u}\left(x-x_{0}\right) e^{\left(i V_{2} / 2 K\right)\left(x-x_{0}\right)+i \phi_{1}}, \\
v(0, x)= & \widetilde{v}(x) e^{\left(i V_{1} / 2 K\right) x} \\
& +\widetilde{v}\left(x-x_{0}\right) e^{\left(i V_{2} / 2 K\right)\left(x-x_{0}\right)+i \phi_{2}},
\end{aligned}
$$

where the initial separation between the two coupled-mode solitons with frequencies $V_{i} / 2 K, i=1,2$, is $x_{0}=15$, and $V_{1}=$ 1 and $V_{2}=-1$ are the differing velocities of the two solitary waves produced by this initial condition. Furthermore, $\phi_{1}$ and $\phi_{2}$ are the initial phases of the left-going pulses. Here $\widetilde{u}$ and $\widetilde{v}$ are a coupled-mode solution of (18) obtained by using Newton's iteration with $K=0.5, \sigma_{1}=\sigma_{2}=0, \alpha=1.5, a=c=$ $1, b=d=2 / 3, e=1 / 3$, and initial values $u_{0}(x)=\operatorname{sech}(\xi-35)$

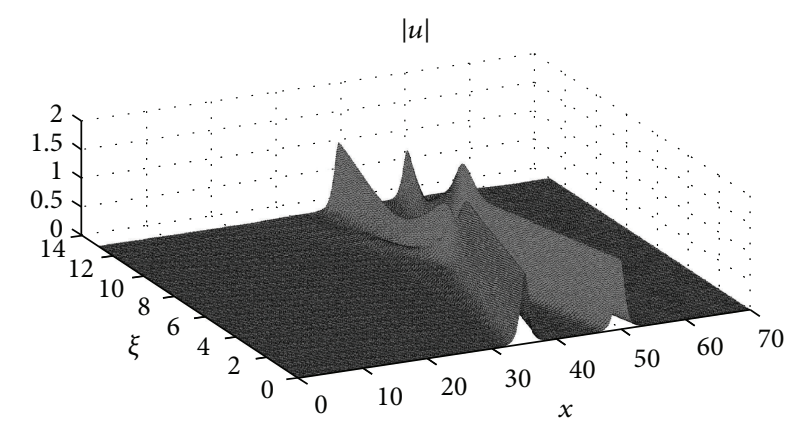

FIGURE 15: Collision of coupled-mode solitons with $\phi_{1}=\pi$ and $\phi_{2}=$ 0 .

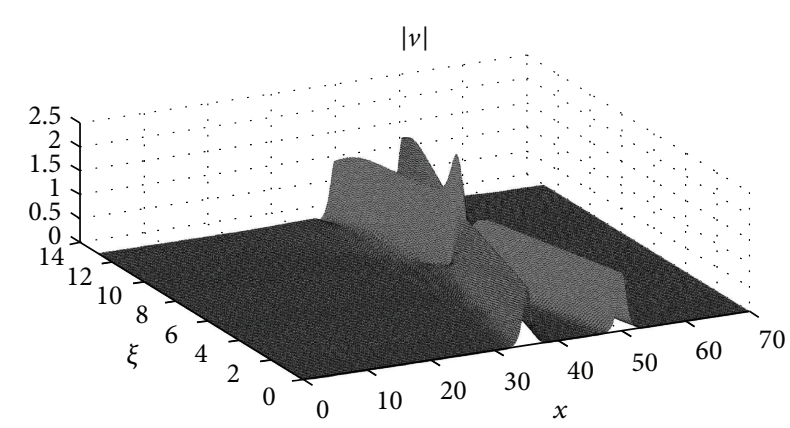

FIGURE 16: Collision of coupled-mode solitons with $\phi_{1}=\pi$ and $\phi_{2}=$ 0 .

and $v_{0}(x)=\operatorname{sech}(\xi-35)$. The numerical parameters for computing the evolution of these pulses with respect to the variable $\xi$ are $L=70$ (length of the spatial computational domain), $\Delta t=1 e-3$ (time step size), and $N=2^{10}$ (number of FFT points). In Figures 11 and 12 we see the collision of the coupled-mode solitons given in (116) with $\phi_{1}=\phi_{2}=$ 0 , and in Figures 13 and 14 are the corresponding contour plots. We observe that the two solitons propagate with the expected velocities $V_{1}=1$ and $V_{2}=-1$, and they elastically (lose little energy) pass through one another; the emergent solitons appear to be unaltered (with approximately the same amplitude and velocity) after the collision. In Figures 15, 16, 17 , and 18 , we repeat the previous numerical simulation to illustrate the collision of the coupled-mode solitons given in (116) when the initial phases are changed to $\phi_{1}=\pi$ and $\phi_{2}=0$. As in the previous computer simulation, the solitons cross each other, but now some exchange of energy is present between the two coupled components $|u(\xi, x)|,|v(\xi, x)|$. As a consequence, we find that they does not maintain their amplitudes after the collision.

\section{Conclusions}

In this paper, using the theory of positive operators in a cone in a Fréchet space [41, 42], we established the existence of vector solitons of the system of coupled Schrödinger equations (1). To achieve this, we extended the techniques in [43] for the case of a family of scalar dispersive equations. We further illustrated the geometry of these solutions by approximating 


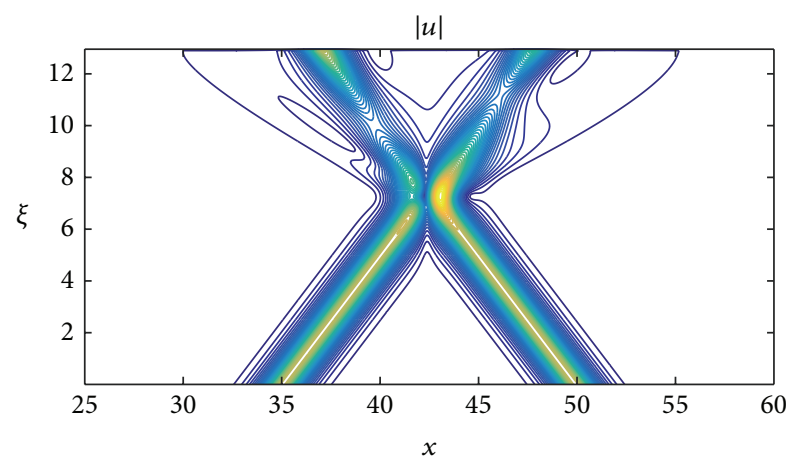

FIGURE 17: Contour plot of the collision of solitons in Figure 15.

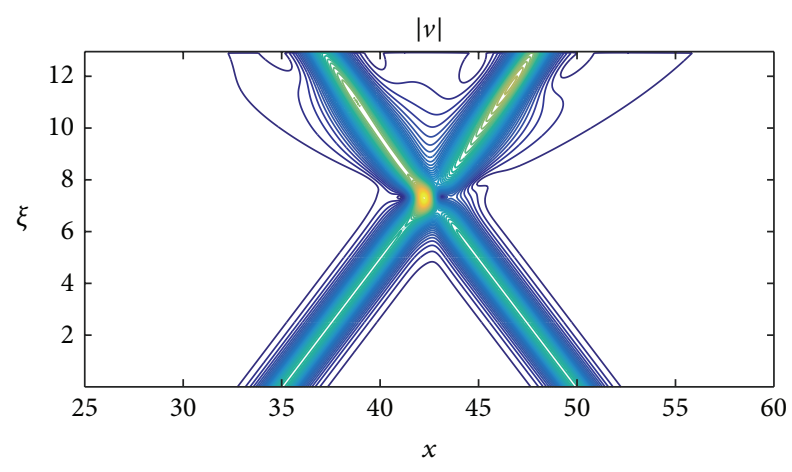

FIGURE 18: Contour plot of the collision of solitons in Figure 16.

them through a numerical solver, which involves a Newtontype iteration together with a spectral discretization for the spatial variable. Our numerical simulations give evidence on the existence of vector solitons for the two parameter regimes analyzed, in accordance with the theory presented. Further research is needed to study other issues of great interest, such as orbital stability under small initial disturbances of vector solitons and relationship between periodic and nonperiodic finite-energy solutions of system (1).

\section{Competing Interests}

The author declares that there are no competing interests regarding the publication of this paper.

\section{Acknowledgments}

This research was supported by Universidad del Valle, Calle 13, No. 100-00, Cali, Colombia, under the research project C.I. 71020 .

\section{References}

[1] J. Catani, L. De Sarlo, G. Barontini, F. Minardi, and M. Inguscio, "Degenerate Bose-Bose mixture in a three-dimensional optical lattice," Physical Review A, vol. 77, no. 1, Article ID 011603, 2008.

[2] P. Maddaloni, M. Modugno, C. Fort, F. Minardi, and M. Inguscio, "Collective oscillations of two colliding Bose-Einstein condensates," Physical Review Letters, vol. 85, no. 12, pp. 24132417, 2000.
[3] M. R. Matthews, B. P. Anderson, P. C. Haljan, D. S. Hall, C. E. Wieman, and E. A. Cornell, "Vortices in a bose-einstein condensate," Physical Review Letters, vol. 83, no. 13, pp. 24982501, 1999.

[4] M. R. Matthews, B. P. Anderson, P. C. Haljan et al., "Watching a superfluid untwist itself: recurrence of rabi oscillations in a Bose-Einstein condensate," Physical Review Letters, vol. 83, no. 17, pp. 3358-3361, 1999.

[5] V. A. Brazhnyi and V. V. Konotop, "Stable and unstable vector dark solitons of coupled nonlinear Schrödinger equations: application to two-component Bose-Einstein condensates," Physical Review E, vol. 72, no. 2, Article ID 026616, 9 pages, 2005.

[6] B. Deconinck, P. G. Kevrekidis, H. E. Nistazakis, and D. J. Frantzeskakis, "Linearly coupled Bose-Einstein condensates: from Rabi oscillations and quasiperiodic solutions to oscillating domain walls and spiral waves," Physical Review A: Atomic, Molecular, and Optical Physics, vol. 70, Article ID 063605, 2004.

[7] J. J. García-Ripoll, J. I. Cirac, J. Anglin, V. M. Pérez-García, and P. Zoller, "Spin monopoles with Bose-Einstein condensates," Physical Review A: Atomic, Molecular, and Optical Physics, vol. 61, no. 5, article 053609, 2000.

[8] K. Kasamatsu and M. Tsubota, "Modulation instability and solitary-wave formation in two-component Bose-Einstein condensates," Physical Review A, vol. 74, no. 1, Article ID 013617, 2006.

[9] A. Zafrany, B. A. Malomed, and I. M. Merhasin, "Solitons in a linearly coupled system with separated dispersion and nonlinearity," Chaos, vol. 15, article 037108, 2005.

[10] Y. Kivshar and G. P. Agrawal, Optical Solitons: From Fiber to Photonic Crystals, Academic Press, 2003.

[11] Y. V. Kartashov, B. A. Malomed, V. A. Vysloukh, and L. Torner, "Vector solitons in nonlinear lattices," Optics Letters, vol. 34, no. 23, pp. 3625-3627, 2009.

[12] L. Pitaevskii and S. Stringari, Bose-Einstein Condensation, vol. 116, Oxford University Press, Oxford, UK, 2003.

[13] C. J. Pethick and H. Smith, Bose-Einstein Condensation in Dilute Gases, Cambridge University Press, Cambridge, UK, 2002.

[14] E. Timmermans, P. Tommasini, M. Hussein, and A. Kerman, "Feshbach resonances in atomic Bose-Einstein condensates," Physics Report, vol. 315, no. 1-3, pp. 199-230, 1999.

[15] E. Timmermans, P. Tommasini, R. Côté, M. Hussein, and A. Kerman, "Rarified liquid properties of hybrid atomic-molecular Bose-Einstein condensates," Physical Review Letters, vol. 83, no. 14, pp. 2691-2694, 1999.

[16] M. I. Rodas-Verde, H. Michinel, and V. M. Pérez-García, “Controllable soliton emission from a Bose-Einstein condensate," Physical Review Letters, vol. 95, no. 15, Article ID 153903, 2005.

[17] G. Theocharis, P. Schmelcher, P. G. Kevrekidis, and D. J. Frantzeskakis, "Matter-wave solitons of collisionally inhomogeneous condensates," Physical Review A, vol. 72, no. 3, Article ID 033614, 2005.

[18] M. T. Primatarowa, K. T. Stoychev, and R. S. Kamburova, "Interaction of solitons with extended nonlinear defects," Physical Review E, vol. 72, no. 3, Article ID 036608, 2005.

[19] F. K. Abdullaev and J. Garnier, "Propagation of matter-wave solitons in periodic and random nonlinear potentials," Physical Review A, vol. 72, Article ID 061605, 2005.

[20] J. Garnier and F. K. Abdullaev, "Transmission of matter-wave solitons through nonlinear traps and barriers," Physical Review A-Atomic, Molecular, and Optical Physics, vol. 74, article 013604, 2006. 
[21] A. V. Carpentier, H. Michinel, M. I. Rodas-Verde, and V. M. Pérez-García, "Analysis of an atom laser based on the spatial control of the scattering length," Physical Review A-Atomic, Molecular, and Optical Physics, vol. 74, article 013619, 2006.

[22] H. Sakaguchi and B. Malomed, "Two-dimensional solitons in the Gross-Pitaevskii equation with spatially modulated nonlinearity," Physical Review E, vol. 73, no. 2, Article ID 026601, 2006.

[23] G. Dong and B. Hu, "Management of Bose-Einstein condensates by a spatially periodic modulation of the atomic s-wave scattering length," Physical Review A, vol. 75, no. 1, Article ID 013625, 2007.

[24] P. Niarchou, G. Theocharis, P. G. Kevrekidis, P. Schmelcher, and D. J. Frantzeskakis, "Soliton oscillations in collisionally inhomogeneous attractive Bose-Einstein condensates," Physical Review A-Atomic, Molecular, and Optical Physics, vol. 76, article 023615, 2007.

[25] S. V. Manakov, "On the theory of two dimensional stationary self-focusing of electromagnetic waves," Zhurnal Eksperimental'noi i Teroreticheskoi Fiziki, vol. 65, pp. 505-516, 1973.

[26] A. C. Scott, "Launching a Davydov soliton: I. Soliton analysis," Physica Scripta, vol. 29, no. 3, pp. 279-283, 1984.

[27] Z. Y. Yan, "Vector financial rogue waves," Physics Letters A, vol. 375, no. 48, pp. 4274-4279, 2011.

[28] K. Dysthe, H. E. Krogstad, and P. Muller, "Oceanic rogue waves," Annual Review of Fluid Mechanics, vol. 40, pp. 287-310, 2008.

[29] T. Ueda and W. L. Kath, "Dynamics of coupled solitons in nonlinear optical fibers," Physical Review A, vol. 42, no. 1, pp. 563-571, 1990.

[30] C. R. Menyuk, "Nonlinear pulse propagation in birefringent optical fibers," IEEE Journal of Quantum Electronics, vol. 23, no. 2, pp. 174-176, 1987.

[31] C. R. Menyuk, "Pulse propagation in an elliptically birefringent Kerr medium," IEEE Journal of Quantum Electronics, vol. 25, no. 12, pp. 2674-2682, 1989.

[32] G. P. Agrawal, Nonlinear Fiber Optics, Academic Press, New York, NY, USA, 3rd edition, 2001.

[33] S. G. Evangelides Jr., L. F. Mollenauer, J. P. Gordon, and N. S. Bergano, "Polarization multiplexing with solitons," Journal of Lightwave Technology, vol. 10, no. 1, pp. 28-35, 1992.

[34] W. B. Cardoso, A. T. Avelar, D. Bazeia, and M. S. Hussein, "Solitons of two-component Bose-Einstein condensates modulated in space and time," Physics Letters, Section A: General, Atomic and Solid State Physics, vol. 374, no. 23, pp. 2356-2360, 2010.

[35] L. Han, Y. Huang, and H. Liu, "Solitons in coupled nonlinear Schrödinger equations with variable coefficients," Communications in Nonlinear Science and Numerical Simulation, vol. 19, no. 9, pp. 3063-3073, 2014.

[36] M. Theis, G. Thalhammer, K. Winkler et al., "Tuning the scattering length with an optically induced feshbach resonance," Physical Review Letters, vol. 93, article 123001, 2004.

[37] J. C. Muñoz Grajales and J. C. Quiceno, "Modulation instability in nonlinear propagation of pulses in optical fibers," Applied Mathematics and Computation, vol. 221, pp. 177-191, 2013.

[38] J. Belmonte-Beitia, V. M. Pérez-García, and V. Brazhnyi, "Solitary waves in coupled nonlinear Schrödinger equations with spatially inhomogeneous nonlinearities," Communications in Nonlinear Science and Numerical Simulation, vol. 16, no. 1, pp. 158-172, 2011.

[39] J. Belmonte-Beitia, V. M. Pérez-García, and P. J. Torres, "Solitary waves for linearly coupled nonlinear Schrödinger equations with inhomogeneous coefficients," Journal of Nonlinear Science, vol. 19, no. 4, pp. 437-451, 2009.
[40] R. Radha, P. S. Vinayagam, J. B. Sudharsan, and B. A. Malomed, "Persistent bright solitons in sign-indefinite coupled nonlinear Schrödinger equations with a time-dependent harmonic trap," Communications in Nonlinear Science and Numerical Simulation, vol. 31, no. 1-3, pp. 30-39, 2016.

[41] M. A. Krasnosel'skii, Positive Solutions of Operators Equations, L.F. Boron: P. Noordhoff Ltd, Groningen, Netherlands, 1964.

[42] M. A. Krasnosel'skii, Topological Methods in the Theory of Nonlinear Integral Equations, Pergamon Press, New York, NY, USA, 1964.

[43] T. B. Benjamin, J. L. Bona, and D. K. Bose, "Solitary-wave solutions of nonlinear problems," Philosophical Transactions of the Royal Society of London A, vol. 331, no. 1617, pp. 195-244, 1990.

[44] H. Chen, "Existence of periodic travelling-wave solutions of nonlinear, dispersive wave equations," Nonlinearity, vol. 17, no. 6, pp. 2041-2056, 2004.

[45] H. Chen, M. Chen, and N. V. Nguyen, "Cnoidal wave solutions to Boussinesq systems," Nonlinearity, vol. 20, no. 6, pp. $1443-$ 1461, 2007.

[46] W. Rudin, Functional Analysis, McGraw-Hill, New York, NY, USA, 1973.

[47] A. Granas, "The Leray-Schauder index and fixed point theory for arbitrary ANRs," Bulletin de la Société Mathématique de France, vol. 100, pp. 209-228, 1972.

[48] J. B. Conway, Functions of One Complex Variable, vol. 11 of Graduate Texts in Mathematics, Springer, New York, NY, USA, 2nd edition, 1978

[49] F. A. Pipicano and J. C. Muñoz Grajales, "Existence of periodic travelling wave solutions for a regularized Benjamin-Ono system," Journal of Differential Equations, vol. 259, no. 12, pp. 7503$7528,2015$. 


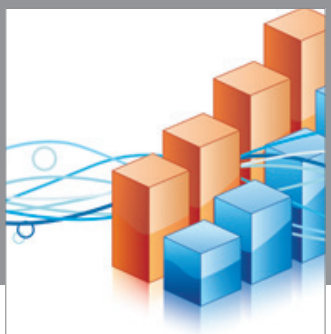

Advances in

Operations Research

vatem alat4

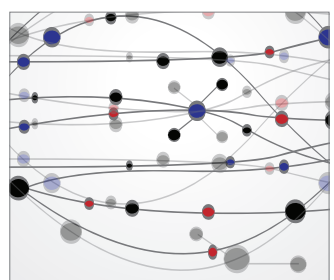

\section{The Scientific} World Journal
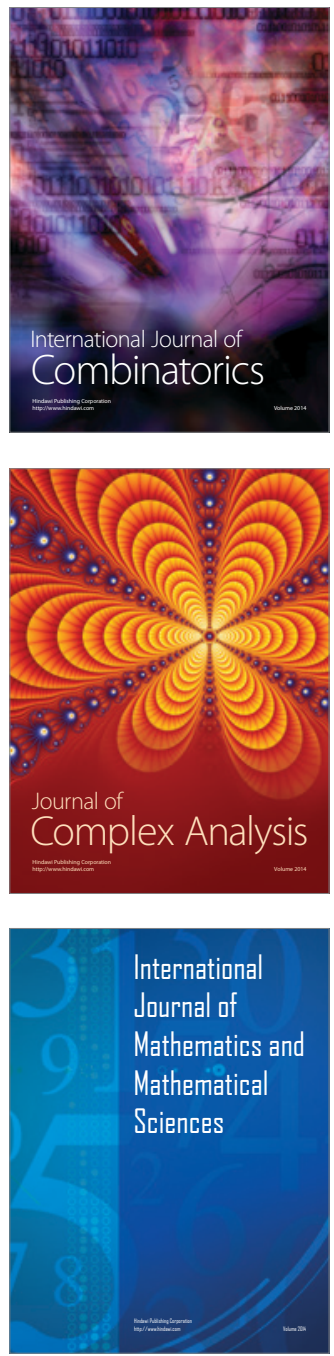
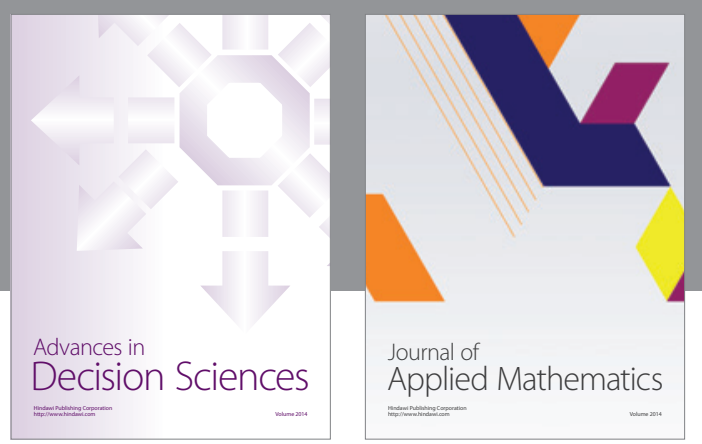

Algebra

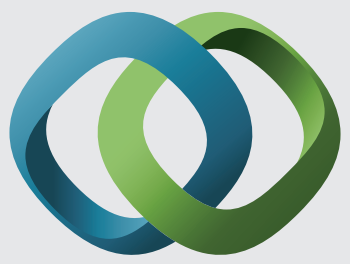

\section{Hindawi}

Submit your manuscripts at

http://www.hindawi.com
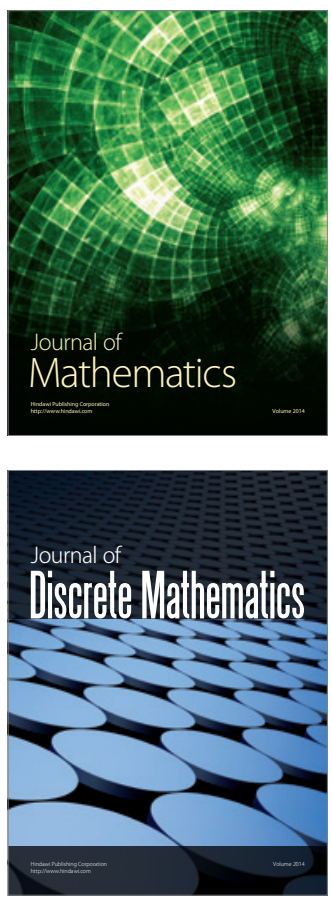

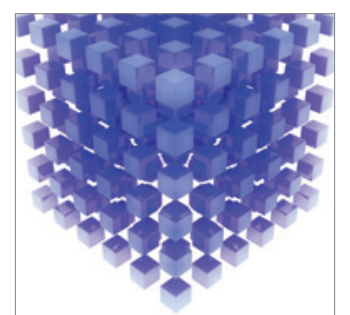

Mathematical Problems in Engineering
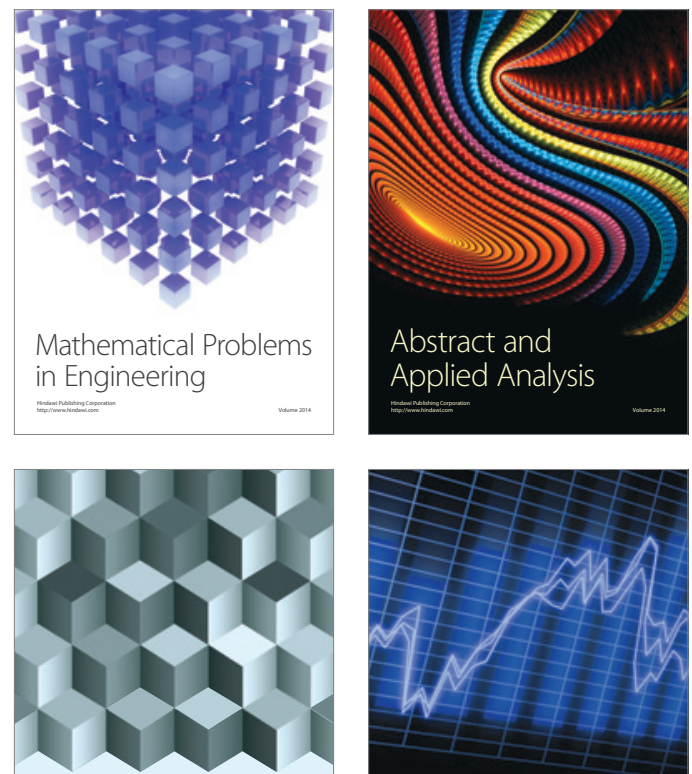

Journal of

Function Spaces

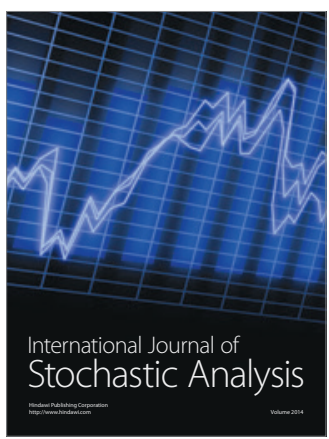

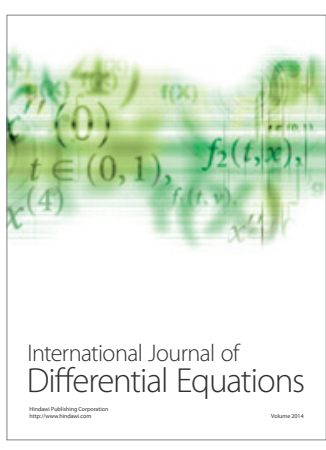
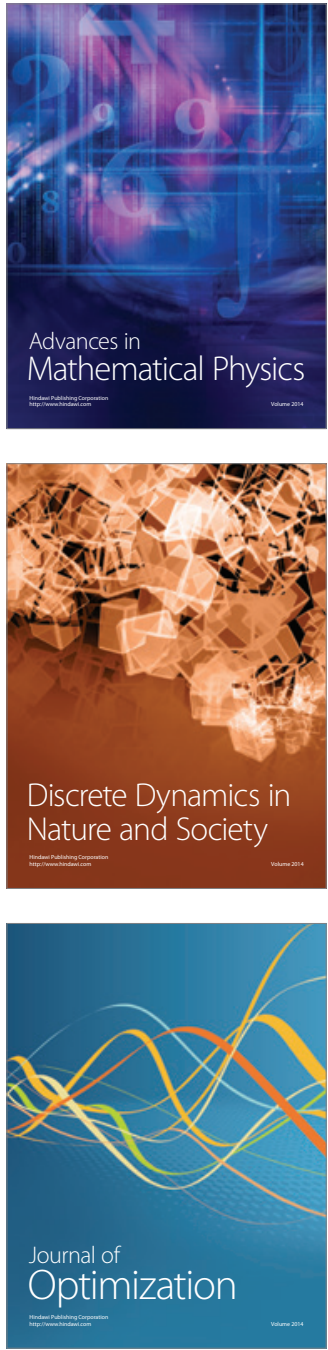